\title{
Association between Metabolites and the Risk of Lung Cancer: A Systematic Literature Review and Meta-Analysis of Observational Studies
}

\author{
Kian Boon Lee ${ }^{1}$, Lina Ang ${ }^{2}$, Wai-Ping Yau ${ }^{1}$ and Wei Jie Seow ${ }^{2,3, *(D)}$ \\ 1 Department of Pharmacy, Faculty of Science, National University of Singapore, Singapore 117543, Singapore; \\ lee_kian_boon@u.nus.edu (K.B.L.); phaywp@nus.edu.sg (W.-P.Y.) \\ 2 Saw Swee Hock School of Public Health, National University of Singapore and National University Health \\ System, Singapore 117549, Singapore; ephanli@nus.edu.sg \\ 3 Department of Medicine, Yong Loo Lin School of Medicine, National University of Singapore and National \\ University Health System, Singapore 119228, Singapore \\ * Correspondence: ephswj@nus.edu.sg; Tel.: +65-6601-1243; Fax: +65-6779-1489
}

Received: 8 July 2020; Accepted: 24 August 2020; Published: 5 September 2020

\begin{abstract}
Globally, lung cancer is the most prevalent cancer type. However, screening and early detection is challenging. Previous studies have identified metabolites as promising lung cancer biomarkers. This systematic literature review and meta-analysis aimed to identify metabolites associated with lung cancer risk in observational studies. The literature search was performed in PubMed and EMBASE databases, up to 31 December 2019, for observational studies on the association between metabolites and lung cancer risk. Heterogeneity was assessed using the $\mathrm{I}^{2}$ statistic and Cochran's Q test. Meta-analyses were performed using either a fixed-effects or random-effects model, depending on study heterogeneity. Fifty-three studies with 297 metabolites were included. Most identified metabolites (252 metabolites) were reported in individual studies. Meta-analyses were conducted on 45 metabolites. Five metabolites (cotinine, creatinine riboside, $\mathrm{N}$-acetylneuraminic acid, proline and r-1,t-2,3,c-4-tetrahydroxy-1,2,3,4-tetrahydrophenanthrene) and five metabolite groups (total 3-hydroxycotinine, total cotinine, total nicotine, total 4-(methylnitrosamino)-1(3-pyridyl)-1-butanol (sum of concentrations of the metabolite and its glucuronides), and total nicotine equivalent (sum of total 3-hydroxycotinine, total cotinine and total nicotine)) were associated with higher lung cancer risk, while three others (folate, methionine and tryptophan) were associated with lower lung cancer risk. Significant heterogeneity was detected across most studies. These significant metabolites should be further evaluated as potential biomarkers for lung cancer.
\end{abstract}

Keywords: biomarkers; lung cancer; metabolomics; meta-analysis; systematic literature review

\section{Introduction}

Lung cancer is the most common form of malignancy worldwide, particularly in men, with high mortality and morbidity rates [1]. Based on histology, lung cancers are broadly classified into two major subtypes: small-cell lung cancer (SCLC) and non-small-cell lung cancer (NSCLC) [2]. The latest report by the World Health Organization identified lung cancer as the most frequent type of cancer in terms of both incidence and mortality, with 2.1 million new cases and 1.8 million deaths occurring worldwide in 2018 [1]. Lung cancer trends are strongly associated with tobacco consumption patterns [3-5]. Currently, lung cancer incidence and mortality rates are declining in the more developed countries $[3,4]$, which may partially be attributed to the lower prevalence of tobacco smoking [6]. However, lung cancer trends are increasing in Asia and Africa, which is associated with increasing prevalence of tobacco smoking $[3,7]$. 
Although lung cancer is a deadly disease, it can be treated if detected early. The five-year survival rates for localized lung cancer was $56 \%$, as compared with $5 \%$ for metastasized lung cancer [8]. However, despite efforts in lung cancer research, early detection and diagnosis remain as major challenges [9]. A considerable proportion of patients present with late-stage lung cancer at diagnosis (46.8-61.2\% for NSCLC and 61.3-82\% for SCLC) [10]. Considering the high mortality rate of late-stage lung cancer and the difficulty of early diagnosis, identifying potential biomarkers for the detection of early-stage lung cancer is paramount.

In recent years, the use of metabolomics, which is the study of metabolites in biological specimens [11], in lung cancer research has been a subject of great interest. Compared to genomics, transcriptomics and proteomics, which focus on the upstream processes of metabolism, metabolomics directly measures the metabolic profile of an organism, thereby providing a more precise method to detect changes in metabolism [12]. Since malignant cells, including lung cancer cells, have substantially altered genome and metabolism [13-16], the identification of metabolic changes is a potentially viable strategy for elucidating lung cancer etiology and identifying potential biomarkers.

Numerous studies have explored the association between metabolite levels and lung cancer risk [17-21]. However, most studies have been small-scale, with varying direction and strengths of association across studies. Furthermore, the type of biological samples used have been inconsistent across studies. While earlier reviews on this topic have identified three major classes of metabolites (namely amino acids, fatty acids/lipids and metabolites involved in cellular energy production) to be associated with lung cancer [22,23], to date, the association between levels of each metabolite and lung cancer risk has not been quantitatively evaluated. It is worthwhile to examine both alterations in levels of cellular metabolites as well as smoking-related metabolites. Changes in cellular metabolite levels might be related to intrinsic body alterations, while changes in smoking-related metabolites might be a consequence of patient smoking and other environmental exposures. Therefore, a need to systematically review and quantitatively synthesize the results from all pertinent studies is warranted, in order to identify potential biomarkers for validation studies in the future.

In this systematic literature review and meta-analysis, we aimed to provide a review of the current understanding of the association between metabolites and lung cancer risk based on available evidence from published observational studies.

\section{Methods}

\subsection{Literature Search Strategy}

This systematic literature review was performed based on the Preferred Reporting Items for Systematic Reviews and Meta-analyses (PRISMA) guidelines [24] (List S2). PubMed and EMBASE databases were searched from inception to 31 December 2019. The search terms used included "metabolites", "metabolomics", "lung cancer" and its variants (Table S1). Titles and abstracts of searched articles were screened by two reviewers before retrieving full texts of potentially relevant articles for further evaluation. One reviewer then independently evaluated the full text before making the decision to include the study. The reference lists of included articles were further hand-searched to identify additional relevant articles.

\subsection{Study Selection Criteria}

Studies were included if the following inclusion criteria were met: (1) were observational studies; (2) reported at least one metabolite; and, reported either the (3a) estimated hazard ratio (HR)/odds ratio $(\mathrm{OR})$ /risk ratio (RR) for the association between levels of metabolite and any type of lung cancer; or, $(3 b)$ actual metabolite concentrations for lung cancer patients and controls. If more than one report were published using data from the same study, we included either the report with the most detailed information, or the most recent report, in the case where the reports provided a similar level of details. 
Studies were excluded if they met any of the following exclusion criteria: (1) were non-clinical studies or randomized controlled trials, case reports, case series, reviews or conference abstracts; (2) reported on metastasized lung cancer or recurrent cancer only; (3) included cancer patients who were already on treatment prior to recruitment; (4) included controls with another disease state for non-self-controlled studies; (5) did not provide sufficient data for analysis; or, (6) were not published in English or Chinese.

\subsection{Data Extraction}

For studies that met the eligibility criteria, one reviewer performed data extraction using a standardized form. Data extracted from each study were study design, characteristics of study participants, type of metabolites, type and stage of lung cancer (if reported), type of biological samples used and the number of participants for each metabolite and outcome. For studies that reported the association between levels of metabolite and lung cancer (hereafter termed as categorical studies), the HR/OR/RR and 95\% confidence interval (CI) between the highest and lowest category from the models that adjusted for the most covariates were extracted. For studies that reported the concentration of metabolites in lung cancer patients and controls (hereafter termed as concentration studies), the mean and standard deviation (SD) of metabolite concentration were extracted.

For studies that reported the relevant data in only graphical form [25,26], the data were estimated based on the graphical data presented.

One study [27] presented data that were partially reported in a previous study [28]. For this study [27], only the data that were not previously reported were extracted for further analysis.

One study included recurrent cancer cases in their pool of participants [29]. Since the study reported sufficient data to exclude recurrent cases, the mean and SD were recalculated with the recurrent cases excluded.

For one study that reported standard error (SE) instead of SD [30], SD was calculated as:

$$
S D=S E \times \sqrt{n}
$$

where $n$ is the number of study participants.

One study reported the concentration of metabolites in $\mu \mathrm{g} / \mathrm{L}$ [31]. The data were transformed into $\mu \mathrm{mol} / \mathrm{L}$ using the following formulae:

$$
\begin{gathered}
\text { Mean }=\frac{\text { Mean }_{\mu g}}{M_{r}}, \\
S D=\frac{S D_{\mu g}}{M_{r}},
\end{gathered}
$$

where Mean $_{\mu g}, S D_{\mu g}$ is the mean and SD (reported in $\mu \mathrm{g} / \mathrm{L}$ ), and $M_{r}$ is the molecular weight of the metabolite. The molecular weights of the metabolites were retrieved from the Kyoto Encyclopedia of Genes and Genomes (KEGG) database [32].

For studies that reported data for every participant individually [33,34], the mean and SD were calculated as:

$$
\begin{gathered}
\text { Mean }=\frac{\sum c_{i}}{n}, \\
S D=\sqrt{\frac{\sum\left(c_{i}-\text { Mean }\right)^{2}}{n-1}},
\end{gathered}
$$

where $c_{i}$ is the concentration of metabolite for each participant and $n$ is the number of study participants. 
For studies that reported their data as either median, first and third quartiles [25,35] or median and range [36] in lieu of mean and SD, we estimated the mean and SD using the following formulae [37]:

$$
\begin{gathered}
\text { Mean }=\frac{q_{1}+q_{2}+q_{3}}{3}, \\
S D=\frac{q_{3}-q_{1}}{2 \times Z_{\text {inv }}\left(\frac{0.75 n-0.125}{n+0.25}\right)} \\
\text { Mean }=\frac{m+2 q_{2}+M}{4}, \\
S D=\frac{M-m}{2 \times Z_{\text {inv }}\left(\frac{n-0.375}{n+0.25}\right)}
\end{gathered}
$$

where $q_{1}, q_{2}, q_{3}$ are the first, second and third quartiles, $m, M$ are the minimum and maximum, $n$ is the number of study participants and $Z_{i n v}$ is the inverse function of the standard normal distribution.

\subsection{Assessment of Methodological Quality}

The nine-point Newcastle-Ottawa Scale (NOS) was used to evaluate the methodological quality of observational studies in terms of three broad categories: selection of study groups; comparability of study groups; and the determination of either the exposure (for case-control studies) or outcome (for cohort studies) of interest [38]. Scoring was performed independently by two reviewers (K.B.L and L.A.) and discrepancies in quality scores were resolved by discussion and re-examination of the full-text articles. Any outstanding discrepancies were resolved by discussion with another investigator (W.J.S.).

For self-controlled case-control studies (4 studies) [33,39-41], we used a modified version of the NOS by excluding the question on the definition of controls, as the criterion was not relevant to the studies. The overall quality scores for these studies were then scaled to a maximum score of nine points, in order to facilitate comparison across all studies.

For articles that reported data from more than one cohort (2 studies) [42,43], we evaluated the quality of each cohort separately and assigned an overall score by taking the mean of the scores from the cohorts.

The criterion for defining a high-quality study was neither defined by the original authors of NOS, nor was it validated in the current literature. Nevertheless, we classified studies as low, moderate or high quality based on the scores of 0-3 points, 4-6 points or 7-9 points, respectively [44].

\subsection{Statistical Analysis}

Data were grouped based on the metabolite and the type of biological sample used in the quantification of the metabolite. Meta-analyses were conducted if the metabolite from a biological sample type was reported in at least two studies. For categorical studies, the reported strength of association and the $95 \%$ CI were natural log-transformed before statistical analyses were performed [45]. The results were presented as estimated effect size and 95\% CI. For concentration studies, the results were presented as weighted mean difference (WMD) and 95\% CI. Stratified analyses were performed based on smoking status and gender.

Statistical heterogeneity across studies and subgroups was assessed using the $\mathrm{I}^{2}$ statistic and the p-value from the Cochran's $Q$ test. The random-effects model [46] was used to account for significant heterogeneity across studies if $\mathrm{I}^{2}$ was $>40 \%$ and $\mathrm{p}$-value was $<0.1$, while the fixed-effects model was used to obtain more precise estimates when either condition was not met [45]. The inverse variance approach was used for weighting of the individual studies. All statistical analyses were performed using Stata [47], with a $p$-value $<0.05$ considered as statistically significant. 


\section{Results}

\subsection{Eligible Studies}

The literature search process is shown in Figure 1. Of the 6140 unique articles that were identified based on the literature search strategy, 213 articles were included for full-text review. A total of 53 studies were included in the systematic literature review, of which, 27 studies were categorical studies [42,43,48-72], 24 studies were concentration studies [25-31,33-36,39-41,73-82], and two studies reported both the metabolite concentration and its association with lung cancer risk [83,84]. Most studies (84 studies) with full-texts retrieved for review were subsequently excluded because they did not report sufficient data for further analysis, such as reporting the fold-change in metabolite concentrations between lung cancer patients and controls, instead of actual concentrations of metabolites.

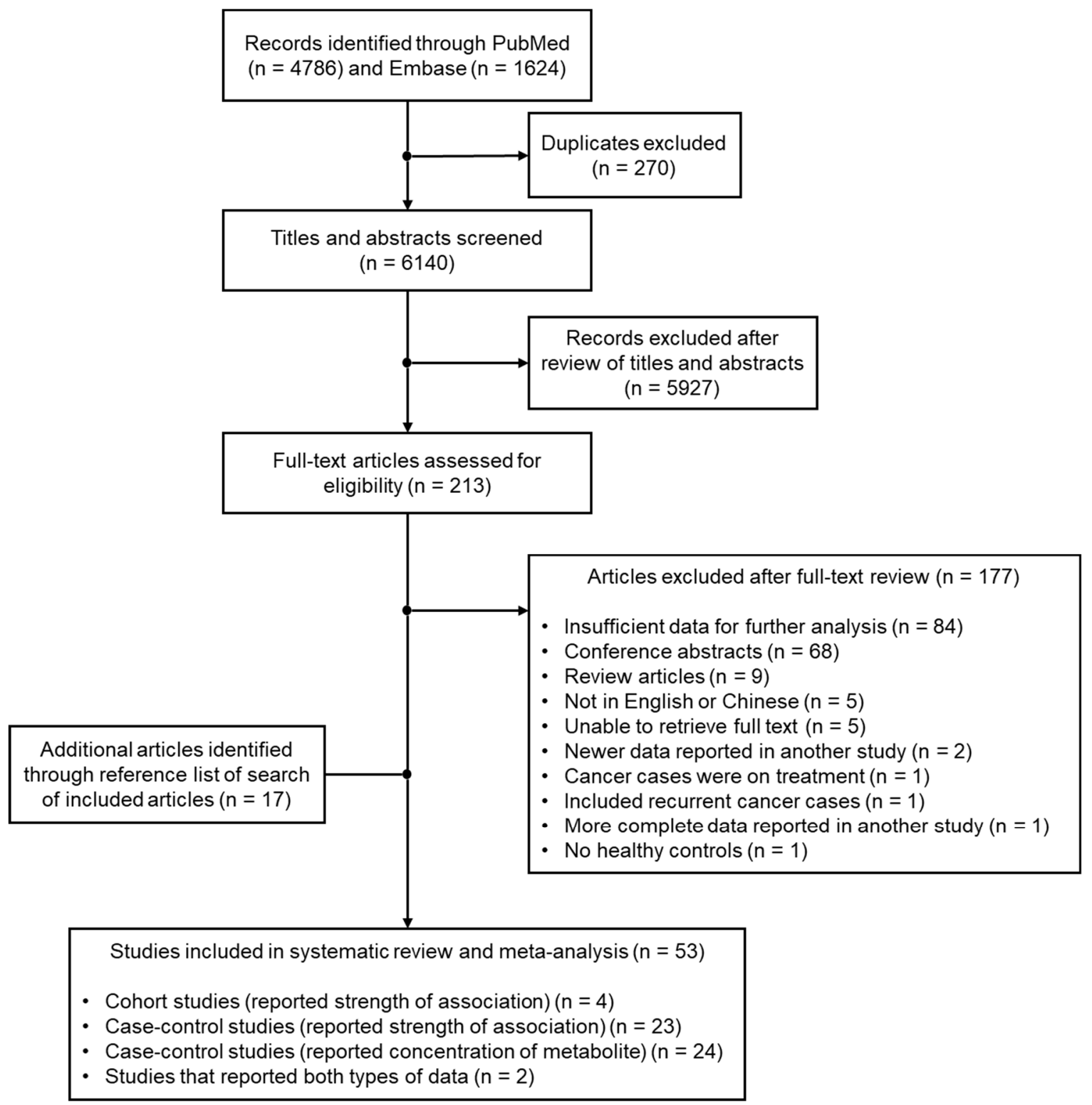

Figure 1. Flowchart of the literature search process and selection of studies. 
Two studies $[85,86]$ were excluded from the review as more recent studies on the same group of participants were published $[28,58,67]$. One study [87] was excluded from the review as the more complete set of data for the same group of participants was published in another study [59].

\subsection{Study Characteristics}

Included studies were published between 1982 and 2019 (Table 1). Thirty-eight studies enrolled participants from both men and women [25-29,33-36,42,43,48,49,53-56,60-62,64,65,67-71,73,74,76-84], three studies included only female participants [50,51,72], and ten studies included only male participants $[30,39,41,52,57-59,63,66,75]$. Two studies did not report the gender of their participants [31,40].

The majority of the studies included participants with any smoking status (33 studies) $[25,27-29,34-$ $36,42,43,48-56,60-62,64,65,68-71,75,78-80,82,84]$. Eight studies included active smokers exclusively [41, $57-59,66,67,76,83]$ and three studies only included participants who had never been smokers $[63,72,77]$. Nine studies did not report the smoking status of their participants $[26,30,31,33,39,40,73,74,81]$.

Most of the studies were conducted in Asia (22 studies) [26-29,31,36,41,52,58-60,63,64,66,67,72, 75-78,81,82], followed by Europe (18 studies) [30,35,42,43,48-51,53-57,61,71,79,80,84] and the USA (7 studies) $[34,39,40,62,65,69,83]$. Two studies involved patient cohorts from the USA, Europe, Asia and Australia $[68,70]$. Four studies did not report the location of their study $[25,33,73,74]$.

Serum samples were the most common biological sample collected in the studies (21 studies) [26,27, $35,36,42,43,48,53,55-57,64,68-70,74,79-81,83,84]$, followed by urine (18 studies) [26,29,50-52,54,58-60, $62,63,65-67,71,72,75,77]$, plasma (11 studies) [25,28,30,31,49,56,68,70,73,78,82], blood (2 studies) [61,76], tumor tissue (3 studies) [33,39,41], and exhaled breath (2 studies) [34,40]. Four studies collected more than one type of biological sample in their study: three studies collected both serum and plasma samples $[56,68,70]$ and one study collected both serum and urine samples [26]. 
Table 1. Characteristics of included studies $(n=53)$.

\begin{tabular}{|c|c|c|c|c|c|c|c|c|c|}
\hline Reference, Year & $\begin{array}{l}\text { No. of Study } \\
\text { Participants } \\
\text { (Case/Control) }\end{array}$ & $\begin{array}{l}\text { Type of } \\
\text { Lung Cancer } \\
\text { Cases (n) }\end{array}$ & $\begin{array}{c}\text { Type of } \\
\text { Biological } \\
\text { Sample }\end{array}$ & $\begin{array}{l}\text { Method Used for } \\
\text { Metabolite } \\
\text { Identification }\end{array}$ & $\begin{array}{c}\text { Level of } \\
\text { Identification } \mathrm{g}\end{array}$ & $\begin{array}{l}\text { Age of Participants } \\
\text { at Recruitment }\end{array}$ & $\begin{array}{c}\text { Gender of } \\
\text { Participants }\end{array}$ & $\begin{array}{l}\text { Location of } \\
\text { Study (Study } \\
\text { Name, If } \\
\text { Applicable) }\end{array}$ & Smoking Status ${ }^{a}$ \\
\hline \multicolumn{10}{|c|}{ Cohort studies reporting the association between exposure to metabolite and lung cancer $(n=4)$} \\
\hline $\begin{array}{c}\text { Kilkkinen et al., } \\
2008 \text { [48] }\end{array}$ & $\begin{array}{c}6937 \\
(122 \text { cases }) \\
\end{array}$ & NR & Serum & $\begin{array}{c}\text { Nicotine Metabolite RIA } \\
\text { kit }\end{array}$ & N.A. & $50.9 \pm 14.9^{b}$ & $3207 \mathrm{M}, 3730 \mathrm{~F}$ & $\begin{array}{l}\text { Finland } \\
\text { (MFhes) }\end{array}$ & $2052 \mathrm{~A}, 4885 \mathrm{~N}$ \\
\hline Afzal et al., 2013 [49] & $\begin{array}{c}9791 \\
\text { (507 cases) }\end{array}$ & NR & Plasma & $\begin{array}{l}\text { DiaSorin Liaison } \\
\text { 25(OH)D TOTAL assay }\end{array}$ & N.A. & $58(48-65)^{c}$ & $4359 \mathrm{M}, 5432 \mathrm{~F}$ & $\begin{array}{l}\text { Copenhagen, } \\
\text { Denmark } \\
\text { (CCHS) }\end{array}$ & $7474 \mathrm{E}, 2317 \mathrm{~N}$ \\
\hline \multirow[t]{2}{*}{$\begin{array}{c}\text { Ordóñez-Mena et al., } \\
2016 \text { [42] }\end{array}$} & $\begin{array}{c}8928 \\
\text { (134 cases) } \\
\end{array}$ & NR & Serum & Immunoassay & N.A. & $63(57-67)^{c}$ & $3545 \mathrm{M}, 5383 \mathrm{~F}$ & $\begin{array}{c}\text { Germany } \\
\text { (ESTHER cohort) }\end{array}$ & NR \\
\hline & $\begin{array}{c}4307 \\
\text { (58 cases) }\end{array}$ & NR & Serum & Immunoassay & N.A. & $62(56-68)^{c}$ & $1553 \mathrm{M}, 2754 \mathrm{~F}$ & $\begin{array}{c}\text { Norway } \\
\text { (TROMS } \varnothing \text { cohort) }\end{array}$ & $\mathrm{N} / \mathrm{F}$ \\
\hline \multirow[t]{2}{*}{ Gao et al., 2019a [43] } & $\begin{array}{c}4345 \\
\text { (39 cases) }\end{array}$ & NR & Serum & d-ROM assay & N.A. & $69(64-74)^{c}$ & $1966 \mathrm{M}, 2379 \mathrm{~F}$ & $\begin{array}{c}\text { Germany } \\
\text { (ESTHER cohort) }\end{array}$ & $\begin{array}{l}335 \mathrm{~A}, 1552 \mathrm{~F}, \\
2374 \mathrm{~N}, 84 \mathrm{U}\end{array}$ \\
\hline & $\begin{array}{c}221 / 1000 \\
\text { (case-cohort study) }\end{array}$ & NR & Serum & d-ROM assay & N.A. & $\begin{array}{c}\text { Ca: } 51(44-56)^{\mathrm{c}} \\
\text { Cohort: } 42(37-50)^{\mathrm{c}}\end{array}$ & $\begin{array}{l}\text { Ca: } 150 \mathrm{M}, 71 \mathrm{~F} \\
\text { Cohort: } 548 \mathrm{M}, \\
\quad 452 \mathrm{~F}\end{array}$ & $\begin{array}{c}\text { Norway } \\
\text { (TROMS } \varnothing \text { cohort) }\end{array}$ & $\begin{array}{c}\text { Ca: } 181 \mathrm{~A}, 28 \mathrm{~F}, \\
11 \mathrm{~N}, 1 \mathrm{U} \\
\text { Cohort: } 443 \mathrm{~A}, \\
245 \mathrm{~F}, 310 \mathrm{~N}, 2 \mathrm{U}\end{array}$ \\
\hline \multicolumn{10}{|c|}{ Case-control studies reporting the association between exposure to metabolite and lung cancer $(\mathrm{n}=23)$} \\
\hline $\begin{array}{l}\text { de Waard et al., } \\
1995 \text { [50] }\end{array}$ & $92 / 305$ & NR & 12H Urine & $\begin{array}{l}\text { Capillary gas } \\
\text { chromatography-mass } \\
\text { spectrometry }\end{array}$ & N.A. & $40-64^{\mathrm{d}}$ & $\mathrm{F}$ & $\begin{array}{c}\text { Utrecht, } \\
\text { Netherlands } \\
\text { (DOM Project) }\end{array}$ & $\begin{array}{l}\text { Ca: 69A, 23P } \\
\text { Co: } 257 A, 191 P\end{array}$ \\
\hline Ellard et al., 1995 [51] & $69 / 255$ & NR & 12H Urine & $\begin{array}{l}\text { Automated colorimetric } \\
\text { method - automated } \\
\text { versions of the manual } \\
\text { direct barbituric acid and } \\
\text { alkaline picrate }\end{array}$ & N.A. & $40-64^{\mathrm{d}}$ & $\mathrm{F}$ & $\begin{array}{l}\text { Utrecht, } \\
\text { Netherlands } \\
\text { (DOM Project) }\end{array}$ & $\begin{array}{l}\text { Ca: } 48 \mathrm{~A}, 21 \mathrm{~N} \\
\text { Co: } 58 \mathrm{~A}, 197 \mathrm{P}\end{array}$ \\
\hline $\begin{array}{l}\text { London et al., } \\
2000[52]\end{array}$ & $232 / 710$ & NR & Urine & HPLC & N.A. & $58.8 \pm 4.8^{\mathrm{b}}$ & M & $\begin{array}{l}\text { Shanghai, China } \\
\text { (SCS) }\end{array}$ & $\begin{array}{c}\text { Ca: } 189 \mathrm{~A}, 19 \mathrm{~F}, \\
24 \mathrm{~N} \\
\text { Co: } 337 \mathrm{~A}, 58 \mathrm{~F}, \\
315 \mathrm{~N}\end{array}$ \\
\hline $\begin{array}{l}\text { Boffetta et al., } \\
2006 \text { [53] }\end{array}$ & $1741 / 1741$ & NR & Serum & Qualitative immunoassay & N.A. & $\begin{array}{l}\text { Adults (Actual age } \\
\text { range reported as } \\
\text { categorical data) }\end{array}$ & $\begin{array}{c}\text { Ca: } 1322 \mathrm{M}, \\
419 \mathrm{~F} \\
\text { Co: } 1322 \mathrm{M}, \\
419 \mathrm{~F} \\
\end{array}$ & Norway & $\begin{array}{c}\text { Ca: 1393A, 96E, } \\
128 \mathrm{~F}, 53 \mathrm{~N}, 71 \mathrm{U} \\
\text { Co: } 727 \mathrm{~A}, 67 \mathrm{E}, \\
411 \mathrm{~F}, 445 \mathrm{~N}, 91 \mathrm{U}\end{array}$ \\
\hline Loft et al., 2007 [54] & $251 / 261$ & $\begin{array}{c}\text { AC (81) } \\
\text { SCLC (55) } \\
\text { SCC (51) } \\
\text { Others (34) }\end{array}$ & Urine & HPLC & N.A. & $50-64^{d}$ & $\begin{array}{c}\text { Ca: } 138 \mathrm{M}, \\
113 \mathrm{~F} \\
\text { Co: } 146 \mathrm{M}, \\
115 \mathrm{~F}\end{array}$ & $\begin{array}{l}\text { Denmark } \\
\text { (DCH Study) }\end{array}$ & $\begin{array}{c}\text { In total, } \\
399 \mathrm{~A}, 94 \mathrm{~F}, 15 \mathrm{~N}\end{array}$ \\
\hline
\end{tabular}


Table 1. Cont.

\begin{tabular}{|c|c|c|c|c|c|c|c|c|c|}
\hline Reference, Year & $\begin{array}{c}\text { No. of Study } \\
\text { Participants } \\
\text { (Case/Control) }\end{array}$ & $\begin{array}{c}\text { Type of } \\
\text { Lung Cancer } \\
\text { Cases (n) }\end{array}$ & $\begin{array}{c}\text { Type of } \\
\text { Biological } \\
\text { Sample }\end{array}$ & $\begin{array}{l}\text { Method Used for } \\
\text { Metabolite } \\
\text { Identification }\end{array}$ & $\begin{array}{c}\text { Level of } \\
\text { Identification } \mathrm{g}\end{array}$ & $\begin{array}{l}\text { Age of Participants } \\
\text { at Recruitment }\end{array}$ & $\begin{array}{c}\text { Gender of } \\
\text { Participants }\end{array}$ & $\begin{array}{c}\text { Location of } \\
\text { Study (Study } \\
\text { Name, If } \\
\text { Applicable) } \\
\end{array}$ & Smoking Status ${ }^{a}$ \\
\hline $\begin{array}{l}\text { Johansson et al., } \\
2010[55]\end{array}$ & $899 / 1815$ & $\begin{array}{c}\text { SCLC (110) } \\
\text { AC (272) } \\
\text { LCC (50) } \\
\text { SCC (200) } \\
\text { Others (267) }\end{array}$ & Serum & $\begin{array}{l}\text { LC-MS/MS, GC-MS/MS } \\
\text { and microbiological assay }\end{array}$ & N.A. & $59(43-73)^{\mathrm{e}}$ & $\begin{array}{c}\text { Ca: } 559 \mathrm{M}, \\
340 \mathrm{~F} \\
\text { Co: } 1126 \mathrm{M}, \\
689 \mathrm{~F}\end{array}$ & $\begin{array}{c}\text { Europe } \\
\text { (EPIC Study) }\end{array}$ & $\begin{array}{c}\text { Ca: } 529 \mathrm{~A}, 260 \mathrm{~F}, \\
96 \mathrm{~N}, 14 \mathrm{U} \\
\mathrm{Co:} 413 \mathrm{~A}, 663 \mathrm{~F}, \\
707 \mathrm{~N}, 32 \mathrm{U}\end{array}$ \\
\hline $\begin{array}{l}\text { Timofeeva et al., } \\
2011 \text { [56] }\end{array}$ & $894 / 1805$ & $\begin{array}{c}\text { SCLC (108) } \\
\text { AC (270) } \\
\text { LCC (50) } \\
\text { SCC (199) } \\
\text { Others/Unknown } \\
(267)\end{array}$ & Plasma/Serum & LC-MS/MS & N.A. & $\begin{array}{l}\text { Adults (Actual age } \\
\text { range reported as } \\
\text { categorical data) }\end{array}$ & $\begin{array}{c}\text { Ca: } 556 \mathrm{M}, \\
338 \mathrm{~F} \\
\text { Co: } 1117 \mathrm{M}, \\
688 \mathrm{~F}\end{array}$ & $\begin{array}{c}\text { Europe } \\
\text { (EPIC Study) }\end{array}$ & $\begin{array}{c}\text { Ca: } 526 \mathrm{~A}, 258 \mathrm{~F}, \\
96 \mathrm{~N} \\
\text { Co: } 409 \mathrm{~A}, 659 \mathrm{~F}, \\
705 \mathrm{~N}\end{array}$ \\
\hline $\begin{array}{l}\text { Weinstein et al., } \\
2011 \text { [57] }\end{array}$ & $500 / 500$ & $\begin{array}{c}\text { SCLC (100) } \\
\text { SCC (179) } \\
\text { AC (73) } \\
\text { Others (148) }\end{array}$ & Fasting serum & $\begin{array}{c}\text { DiaSorin Liaison } \\
\text { 25(OH)D TOTAL assay }\end{array}$ & N.A. & $59(55-62)^{\mathrm{c}}$ & $\mathrm{M}$ & $\begin{array}{l}\text { Finland } \\
\text { (ATBC) }\end{array}$ & A \\
\hline Yuan et al., 2011 [58] & $476 / 476$ & $\begin{array}{c}\text { AC (105) } \\
\text { SCC (153) } \\
\text { SCLC (22) } \\
\text { Others (35) } \\
\text { Unknown (161) }\end{array}$ & Urine & LC-MS/MS, GC-MS/MS & N.A. & $\begin{array}{l}\text { Ca: } 57.4 \pm 5.0^{\mathrm{b}} \\
\text { Co: } 57.2 \pm 4.9^{\mathrm{b}}\end{array}$ & $\mathrm{M}$ & $\begin{array}{l}\text { Shanghai, China } \\
\text { (SCS) }\end{array}$ & A \\
\hline Yuan et al., 2012 [59] & $343 / 392$ & $\begin{array}{c}\text { AC (70) } \\
\text { SCC (104) } \\
\text { SCLC (22) } \\
\text { Others (28) } \\
\text { Unknown (119) }\end{array}$ & Urine & LC-MS/MS, GC-MS/MS & N.A. & NR & $\mathrm{M}$ & $\begin{array}{l}\text { Shanghai, China } \\
\text { (SCS) }\end{array}$ & A \\
\hline Eom et al., 2013 [60] & $35 / 140$ & NR & Urine & HPLC & N.A. & $\begin{array}{l}\text { Ca: } 68.87 \pm 6.86^{\mathrm{b}} \\
\text { Co: } 68.86 \pm 6.85^{\mathrm{b}}\end{array}$ & $\begin{array}{c}\text { Ca: } 27 \mathrm{M}, 8 \mathrm{~F} \\
\text { Co: } 108 \mathrm{M}, 32 \mathrm{~F}\end{array}$ & $\begin{array}{l}\text { South Korea } \\
\text { (KMCC) }\end{array}$ & $\begin{array}{c}\text { Ca: } 28 \mathrm{~A} / \mathrm{F}, 7 \mathrm{~N} \\
\text { Co: } 91 \mathrm{~A} / \mathrm{F}, 48 \mathrm{~N}\end{array}$ \\
\hline $\begin{array}{l}\text { Chuang et al., } \\
2014 \text { [61] }\end{array}$ & $893 / 1748$ & $\begin{array}{l}\text { SCLC (140) } \\
\text { AC (284) } \\
\text { LCC (63) } \\
\text { SCC(198) } \\
\text { Others (208) }\end{array}$ & Blood & LC-MS/MS, GC-MS/MS & N.A. & $59(42-72)^{\mathrm{e}}$ & $\begin{array}{c}\text { Ca: } 556 \mathrm{M}, \\
337 \mathrm{~F} \\
\text { Co: } 1086 \mathrm{M}, \\
662 \mathrm{~F}\end{array}$ & $\begin{array}{c}\text { Europe } \\
\text { (EPIC Study) }\end{array}$ & $\begin{array}{c}\text { Ca: } 526 \mathrm{~A}, 257 \mathrm{~F}, \\
96 \mathrm{~N}, 14 \mathrm{U} \\
\text { Co: } 396 \mathrm{~A}, 648 \mathrm{~F}, \\
674 \mathrm{~N}, 30 \mathrm{U}\end{array}$ \\
\hline Mathe et al., 2014 [62] & $469 / 536$ & NSCLC & Urine & UPLC-ESI-QTOFMS & Level 1 & $\begin{array}{l}\text { Ca: } 66.2^{f} \\
\text { Co: } 66.6^{f}\end{array}$ & $\begin{array}{c}\text { Ca: } 237 \mathrm{M}, \\
232 \mathrm{~F} \\
\text { Co: } 276 \mathrm{M}, \\
260 \mathrm{~F}\end{array}$ & $\begin{array}{l}\text { Greater Baltimore, } \\
\text { Maryland, USA }\end{array}$ & $\begin{array}{c}\text { Ca: } 222 \mathrm{~A}, 214 \mathrm{~F}, \\
33 \mathrm{~N} \\
\text { Co: } 71 \mathrm{~A}, 249 \mathrm{~F} \\
216 \mathrm{~N}\end{array}$ \\
\hline
\end{tabular}


Table 1. Cont.

\begin{tabular}{|c|c|c|c|c|c|c|c|c|c|}
\hline Reference, Year & $\begin{array}{l}\text { No. of Study } \\
\text { Participants } \\
\text { (Case/Control) }\end{array}$ & $\begin{array}{c}\text { Type of } \\
\text { Lung Cancer } \\
\text { Cases (n) }\end{array}$ & $\begin{array}{c}\text { Type of } \\
\text { Biological } \\
\text { Sample }\end{array}$ & $\begin{array}{l}\text { Method Used for } \\
\text { Metabolite } \\
\text { Identification }\end{array}$ & $\begin{array}{c}\text { Level of } \\
\text { Identification } \mathrm{g}\end{array}$ & $\begin{array}{l}\text { Age of Participants } \\
\text { at Recruitment }\end{array}$ & $\begin{array}{l}\text { Gender of } \\
\text { Participants }\end{array}$ & $\begin{array}{c}\text { Location of } \\
\text { Study (Study } \\
\text { Name, If } \\
\text { Applicable) } \\
\end{array}$ & Smoking Status a \\
\hline Yuan et al., 2014 [63] & $82 / 83$ & $\begin{array}{c}\text { SCC (16) } \\
\text { AC (34) } \\
\text { SCLC (2) } \\
\text { Others (9) } \\
\text { Unknown (21) }\end{array}$ & Urine & LC-MS/MS & N.A. & $\begin{array}{l}\text { Ca: } 58.1 \pm 5.2 \mathrm{~b} \\
\text { Co: } 58.0 \pm 5.4^{\mathrm{b}}\end{array}$ & $\mathrm{M}$ & $\begin{array}{c}\text { Shanghai, China } \\
\text { (SCS) }\end{array}$ & $\mathrm{N}$ \\
\hline Wang et al., 2015 [64] & $100 / 100$ & $\begin{array}{c}\text { SCC (35) } \\
\text { AC (51) } \\
\text { Others (14) }\end{array}$ & Serum & $\begin{array}{l}\text { LC-MS/MS and } \\
\text { HPLC }\end{array}$ & N.A. & $\begin{array}{l}\text { Ca: } 57.1 \pm 9.2^{b} \\
\text { Co: } 56.6 \pm 9.2^{b}\end{array}$ & $\begin{array}{l}\text { Ca: } 52 \mathrm{M}, 48 \mathrm{~F} \\
\text { Co: } 51 \mathrm{M}, 49 \mathrm{~F}\end{array}$ & $\begin{array}{l}\text { Changchun, Jilin, } \\
\text { China }\end{array}$ & $\begin{array}{l}\text { Ca: } 48 \mathrm{~A}, 20 \mathrm{~F}, 32 \mathrm{~N} \\
\text { Co: } 9 \mathrm{~A}, 35 \mathrm{~F}, 56 \mathrm{~N}\end{array}$ \\
\hline $\begin{array}{l}\text { Haznadar et al., } \\
2016 \text { [65] }\end{array}$ & $178 / 351$ & $\begin{array}{c}\text { AC (59) } \\
\text { SCC (36) } \\
\text { NSCLC (19) } \\
\text { SCLC (29) } \\
\text { LCC (9) } \\
\text { Others (13) } \\
\text { Unknown (13) }\end{array}$ & Urine & UPLC-MS & Level 1 & $\begin{array}{l}\text { Ca: } 57.7 \pm 8.6^{\mathrm{b}} \\
\text { Co: } 57.3 \pm 8.5^{\mathrm{b}}\end{array}$ & $\begin{array}{c}\text { Ca: } 101 \mathrm{M}, 77 \mathrm{~F} \\
\text { Co: } 194 \mathrm{M}, \\
152 \mathrm{~F}\end{array}$ & $\begin{array}{l}\text { South-eastern } \\
\text { states, USA } \\
\text { (SCCS) }\end{array}$ & $\begin{array}{c}\text { Ca: } 127 \mathrm{~A}, 39 \mathrm{~F}, 7 \mathrm{~N} \\
\text { Co: } 140 \mathrm{~A}, 99 \mathrm{~F} \\
97 \mathrm{~N}\end{array}$ \\
\hline Yuan et al., 2016 [66] & $325 / 356$ & $\begin{array}{c}\text { SCC (102) } \\
\text { AC (80) } \\
\text { SCLC (15) } \\
\text { Others (17) } \\
\text { Unknown (111) }\end{array}$ & Urine & LC-MS/MS, GC-MS/MS & N.A. & $\begin{array}{l}\text { Ca: } 56.7 \pm 4.9^{b} \\
\text { Co: } 56.7 \pm 4.9^{b}\end{array}$ & $\mathrm{M}$ & $\begin{array}{l}\text { Shanghai, China } \\
\text { (SCS) }\end{array}$ & A \\
\hline Yuan et al., 2017 [67] & $197 / 197$ & $\begin{array}{c}\text { AC (51) } \\
\text { SCC (48) } \\
\text { SCLC (25) } \\
\text { Others (49) } \\
\text { Unknown (24) }\end{array}$ & Urine & LC-MS/MS, GC-MS/MS & N.A. & $60.8 \pm 6.2^{b}$ & $\begin{array}{l}\text { Ca: } 165 \mathrm{M}, 32 \mathrm{~F} \\
\text { Co: } 164 \mathrm{M}, 33 \mathrm{~F}\end{array}$ & $\begin{array}{l}\text { Singapore } \\
\text { (SCHS) }\end{array}$ & A \\
\hline Fanidi et al., 2018 [68] & $5364 / 5364$ & $\begin{array}{c}\text { LCC (174) } \\
\text { SCLC (492) } \\
\text { SCC (836) } \\
\text { AC (2056) } \\
\text { Others/Unknown } \\
(1806)\end{array}$ & Plasma/Serum & $\begin{array}{l}\text { LC-MS/MS, GC-MS/MS } \\
\text { and microbiological assay }\end{array}$ & N.A. & $60(44-72)^{\mathrm{e}}$ & $2908 \mathrm{M}, 2456 \mathrm{~F}$ & $\begin{array}{c}\text { Europe, Australia, } \\
\text { China, Singapore, } \\
\text { USA } \\
\text { (LC3) }\end{array}$ & $\begin{array}{l}2519 \mathrm{~A}, 1518 \mathrm{~F} \\
\quad 1327 \mathrm{~N}\end{array}$ \\
\hline $\begin{array}{c}\text { Haznadar et al., } \\
2018 \text { [69] }\end{array}$ & $406 / 437$ & $\begin{array}{c}\text { AC (202) } \\
\text { SCC (108) } \\
\text { NSCLC (96) }\end{array}$ & Serum & UPLC-MS & N.A. & $\begin{array}{l}\text { Ca: } 66.3 \pm 10.0^{\mathrm{b}} \\
\text { Co: } 67.0 \pm 8.9^{\mathrm{b}}\end{array}$ & $\begin{array}{c}\text { Ca: } 214 \mathrm{M}, \\
192 \mathrm{~F} \\
\text { Co: } 234 \mathrm{M}, \\
203 \mathrm{~F}\end{array}$ & $\begin{array}{c}\text { Baltimore, } \\
\text { Maryland, USA }\end{array}$ & $\begin{array}{c}\text { Ca: } 191 \mathrm{~A}, 186 \mathrm{~F}, \\
29 \mathrm{~N} \\
\text { Co: } 52 \mathrm{~A}, 209 \mathrm{~F} \\
176 \mathrm{~N}\end{array}$ \\
\hline
\end{tabular}


Table 1. Cont.

\begin{tabular}{|c|c|c|c|c|c|c|c|c|c|}
\hline Reference, Year & $\begin{array}{l}\text { No. of Study } \\
\text { Participants } \\
\text { (Case/Control) }\end{array}$ & $\begin{array}{l}\text { Type of } \\
\text { Lung Cancer } \\
\text { Cases (n) }\end{array}$ & $\begin{array}{l}\text { Type of } \\
\text { Biological } \\
\text { Sample }\end{array}$ & $\begin{array}{l}\text { Method Used for } \\
\text { Metabolite } \\
\text { Identification }\end{array}$ & $\begin{array}{l}\text { Level of } \\
\text { Identification } \mathrm{g}\end{array}$ & $\begin{array}{l}\text { Age of Participants } \\
\text { at Recruitment }\end{array}$ & $\begin{array}{c}\text { Gender of } \\
\text { Participants }\end{array}$ & $\begin{array}{l}\text { Location of } \\
\text { Study (Study } \\
\text { Name, If } \\
\text { Applicable) }\end{array}$ & Smoking Status a \\
\hline Larose et al., 2018 [70] & $5364 / 5364$ & $\begin{array}{c}\text { LCC (174) } \\
\text { SCLC (492) } \\
\text { SCC (836) } \\
\text { AC (2056) } \\
\text { Others/Unknown } \\
(1806) \\
\end{array}$ & Plasma/Serum & LC-MS/MS & N.A. & $60(44-72)^{\mathrm{e}}$ & $2908 \mathrm{M}, 2456 \mathrm{~F}$ & $\begin{array}{l}\text { Europe, Australia, } \\
\text { China, Singapore, } \\
\text { USA } \\
\text { (LC3) }\end{array}$ & $\begin{array}{l}\text { 2519A, } 1518 \mathrm{~F} \\
\quad 1327 \mathrm{~N}\end{array}$ \\
\hline Gao et al., 2019b [71] & $245 / 735$ & NR & Urine & $\begin{array}{l}\text { Nitrite/nitrate } \\
\text { colorimetric assay }\end{array}$ & N.A. & $62(59-68)^{c}$ & $\begin{array}{c}\text { Ca: } 170 \mathrm{M}, 75 \mathrm{~F} \\
\text { Co: } 509 \mathrm{M}, \\
226 \mathrm{~F}\end{array}$ & $\begin{array}{c}\text { Germany } \\
\text { (ESTHER cohort) }\end{array}$ & $\begin{array}{c}\text { Ca: } 124 \mathrm{~A}, 87 \mathrm{~F}, \\
29 \mathrm{~N} \\
\text { Co: } 365 \mathrm{~A}, 260 \mathrm{~F}, \\
93 \mathrm{~N}\end{array}$ \\
\hline Seow et al., 2019 [72] & $275 / 289$ & $\begin{array}{c}\text { AC (135) } \\
\text { SCC (9) } \\
\text { Others (19) } \\
\text { Unknown (112) }\end{array}$ & Urine & $\begin{array}{l}\text { UPLC-MS and 600-MHz } \\
\text { hydrogen } 1 \text { NMR }\end{array}$ & Level 2 & $\begin{array}{l}\text { Ca: } 61(52-65)^{\mathrm{c}} \\
\text { Co: } 62(53-66)^{\mathrm{c}}\end{array}$ & F & $\begin{array}{l}\text { Shanghai, China } \\
\text { (SWHS) }\end{array}$ & $\mathrm{N}$ \\
\hline \multicolumn{10}{|c|}{ Studies reporting the concentration of metabolites in lung cancer patients and controls $(n=24)$} \\
\hline $\begin{array}{l}\text { Kukreja et al., } \\
1982 \text { [39] }\end{array}$ & 14 (Self-controlled) & $\begin{array}{l}\text { SCC (8) } \\
\text { AC (4) } \\
\text { LCC (2) }\end{array}$ & Tumor tissue & $\begin{array}{c}\text { Silicic acid } \\
\text { chromatography and } \\
\text { radioimmunoassay }\end{array}$ & N.A. & NR & M & $\begin{array}{l}\text { Chicago, Illinois, } \\
\text { USA }\end{array}$ & NR \\
\hline $\begin{array}{l}\text { Hendrick et al., } \\
\quad 1988 \text { [73] }\end{array}$ & 29/18 & $\begin{array}{l}\text { AC/LCC (11) } \\
\text { SCC (9) } \\
\text { SCLC (8) }\end{array}$ & Plasma & Radioimmunoassay & N.A. & $\begin{array}{l}\text { Ca: } 65.4 \pm 7.0^{\mathrm{b}} \\
\text { Co: } 65.5 \pm 10.2^{\mathrm{b}}\end{array}$ & $\begin{array}{l}\text { Ca: } 20 \mathrm{M}, 9 \mathrm{~F} \\
\text { Co: } 11 \mathrm{M}, 7 \mathrm{~F}\end{array}$ & NR & NR \\
\hline Preti et al., 1988 [34] & $10 / 8$ & $\begin{array}{l}\text { SCC (6) } \\
\text { LCC (2) }\end{array}$ & $\begin{array}{l}\text { Exhaled } \\
\text { breath }\end{array}$ & GC-MS & Level 1 & $\begin{array}{l}\text { Ca: } 54-77^{\mathrm{d}} \\
\text { Co: } 57-66^{\mathrm{d}}\end{array}$ & $\begin{array}{l}\text { Ca: } 7 \mathrm{M}, 3 \mathrm{~F} \\
\text { Co: } 4 \mathrm{M}, 4 \mathrm{~F}\end{array}$ & $\begin{array}{c}\text { Pennsylvania, } \\
\text { USA }\end{array}$ & $\begin{array}{c}\text { Ca: } 4 \mathrm{~A}, 6 \mathrm{~F} \\
\text { Co: } 4 \mathrm{~A}, 1 \mathrm{~F}, 3 \mathrm{~N}\end{array}$ \\
\hline $\begin{array}{l}\text { Proenza et al., } \\
2003 \text { [30] }\end{array}$ & $14 / 14$ & NR & $\begin{array}{l}\text { Fasting } \\
\text { plasma }\end{array}$ & HPLC & N.A. & $\begin{array}{l}\text { Ca: } 64.4 \pm 6.0^{\mathrm{b}} \\
\text { Co: } 59.8 \pm 7.9^{\mathrm{b}}\end{array}$ & M & Spain & NR \\
\hline Masri et al., 2005 [40] & $11 / 35$ & NR & $\begin{array}{l}\text { Exhaled } \\
\text { breath }\end{array}$ & $\begin{array}{l}\text { Chemiluminescent } \\
\text { analyzer, amperometric } \\
\text { sensor }\end{array}$ & N.A. & NR & NR & $\begin{array}{l}\text { Cleaveland, Ohio, } \\
\text { USA }\end{array}$ & NR \\
\hline $\begin{array}{l}\text { Gencer et al., } \\
2006 \text { [74] }\end{array}$ & $38 / 26$ & $\begin{array}{l}\text { EC (14) } \\
\text { SCLC (12) } \\
\text { AC (12) }\end{array}$ & Fasting serum & $\begin{array}{l}\text { Technicon RA-XT } \\
\text { autoanalyzer }\end{array}$ & N.A. & $\begin{array}{l}\text { AC: } 54 \pm 12^{\mathrm{b}} \\
\text { EC: } 59.6 \pm 14^{\mathrm{b}} \\
\text { SCLC: } 52 \pm 9^{\mathrm{b}} \\
\text { Co: } 53.2 \pm 12^{\mathrm{b}}\end{array}$ & $\begin{array}{c}\text { AC: } 9 \mathrm{M}, 3 \mathrm{~F} \\
\text { EC: } 11 \mathrm{M}, 3 \mathrm{~F} \\
\text { SCLC: } 10 \mathrm{M}, \\
2 \mathrm{~F} \\
\text { Co: } 21 \mathrm{M}, 5 \mathrm{~F}\end{array}$ & NR & NR \\
\hline
\end{tabular}


Table 1. Cont.

\begin{tabular}{|c|c|c|c|c|c|c|c|c|c|}
\hline Reference, Year & $\begin{array}{l}\text { No. of Study } \\
\text { Participants } \\
\text { (Case/Control) }\end{array}$ & $\begin{array}{l}\text { Type of } \\
\text { Lung Cancer } \\
\text { Cases (n) }\end{array}$ & $\begin{array}{c}\text { Type of } \\
\text { Biological } \\
\text { Sample }\end{array}$ & $\begin{array}{l}\text { Method Used for } \\
\text { Metabolite } \\
\text { Identification }\end{array}$ & $\begin{array}{c}\text { Level of } \\
\text { Identification } \mathrm{g}\end{array}$ & $\begin{array}{l}\text { Age of Participants } \\
\text { at Recruitment }\end{array}$ & $\begin{array}{c}\text { Gender of } \\
\text { Participants }\end{array}$ & $\begin{array}{c}\text { Location of } \\
\text { Study (Study } \\
\text { Name, If } \\
\text { Applicable) } \\
\end{array}$ & Smoking Status ${ }^{a}$ \\
\hline Zhang et al., 2006 [75] & $10 / 12$ & NR & 24H Urine & HPLC and GC-MS/MS & N.A. & $30-70^{\mathrm{d}}$ & $\mathrm{M}$ & Beijing, China & $\begin{array}{l}\text { Ca: } 2 \mathrm{~A}, 8 \mathrm{~F} \\
\text { Co: } 8 \mathrm{~A}, 4 \mathrm{~N}\end{array}$ \\
\hline Esme et al., 2008 [76] & $49 / 20$ & $\begin{array}{l}\text { AC (24) } \\
\text { SCC (21) } \\
\text { LCC (4) }\end{array}$ & Blood & $\begin{array}{l}\text { Spectrophotometric } \\
\text { method }\end{array}$ & N.A. & $\begin{array}{l}\text { Ca: } 57.2 \pm 10.1^{\mathrm{b}} \\
\text { Co: } 52.1 \pm 12.1^{\mathrm{b}}\end{array}$ & $\begin{array}{l}\text { Ca: } 40 \mathrm{M}, 9 \mathrm{~F} \\
\text { Co: } 9 \mathrm{M}, 11 \mathrm{~F}\end{array}$ & Turkey & A \\
\hline Hu et al., 2009 [26] & $30 / 63$ & NSCLC & $\begin{array}{l}\text { Non-fasting } \\
\text { serum \& } \\
\text { Urine }\end{array}$ & $\begin{array}{l}\text { HPLC, amino acid } \\
\text { analyzer }\end{array}$ & N.A. & $\begin{array}{l}\text { Ca: } 59.7 \pm 8.0^{\mathrm{b}} \\
\text { Co: } 67.0 \pm 5.4^{\mathrm{b}}\end{array}$ & $\begin{array}{l}\text { Ca: } 7 \mathrm{M}, 3 \mathrm{~F} \\
\text { Co: } 4 \mathrm{M}, 4 \mathrm{~F}\end{array}$ & Anhui, China & NR \\
\hline $\begin{array}{l}\text { Miyagi et al., } \\
2011 \text { [28] }\end{array}$ & 200/996 & $\begin{array}{c}\text { AC (133) } \\
\text { SCC (35) } \\
\text { SCLC (8) } \\
\text { Others (9) } \\
\text { Unknown (15) }\end{array}$ & $\begin{array}{l}\text { Fasting } \\
\text { plasma }\end{array}$ & $\begin{array}{l}\text { HPLC-electrospray } \\
\text { ionization mass } \\
\text { spectrometry }\end{array}$ & N.A. & $\begin{array}{l}\text { Ca: } 65.0 \pm 10.0^{\mathrm{b}} \\
\text { Co: } 63.2 \pm 9.2^{\mathrm{b}}\end{array}$ & $\begin{array}{c}\text { Ca: } 125 \mathrm{M}, 75 \mathrm{~F} \\
\text { Co: } 635 \mathrm{M} \\
371 \mathrm{~F}\end{array}$ & Japan & $\begin{array}{c}\text { Ca: } 84 \mathrm{~A}, 54 \mathrm{~F}, 60 \mathrm{~N}, \\
2 \mathrm{U} \\
\text { Co: } 137 \mathrm{~A}, 245 \mathrm{~F} \\
536 \mathrm{~N}, 78 \mathrm{U}\end{array}$ \\
\hline Kami et al., 2013 [33] & 9 (Self-controlled) & $\begin{array}{l}\text { AC (3) } \\
\text { SCC (4) } \\
\text { LCC (1) } \\
\text { PC (1) }\end{array}$ & Tumor tissue & $\begin{array}{l}\text { Capillary electrophoresis } \\
\text { time-of-flight mass } \\
\text { spectrometry }\end{array}$ & Level 2 & $56-82^{d}$ & $8 \mathrm{M}, 1 \mathrm{~F}$ & NR & NR \\
\hline Okur et al., 2013 [41] & 15 (Self-controlled) & $\begin{array}{l}\text { AC (3) } \\
\text { EC (12) }\end{array}$ & Tumor tissue & $\begin{array}{l}\text { Chemiluminescence } \\
\text { assay }\end{array}$ & N.A. & $63.6 \pm 9.2^{b}$ & $\mathrm{M}$ & Istanbul, Turkey & A \\
\hline $\begin{array}{c}\text { Shingyogi et al., } \\
2013 \text { [27] }\end{array}$ & $86 / 323^{h}$ & $\begin{array}{c}\text { AC (55) } \\
\text { SCC (12) } \\
\text { Other NSCLC (8) } \\
\text { SCLC (11) } \\
\text { Unknown (3) }\end{array}$ & Fasting serum & $\begin{array}{l}\text { HPLC-electrospray } \\
\text { ionization mass } \\
\text { spectrometry }\end{array}$ & N.A. & $\begin{array}{l}\text { Ca: } 67.8 \pm 8.2^{b} \\
\text { Co: } 61.9 \pm 6.0^{b}\end{array}$ & $\begin{array}{l}\text { Ca: } 68 \mathrm{M}, 18 \mathrm{~F} \\
\text { Co: } 263 \mathrm{M}, 60 \mathrm{~F}\end{array}$ & Japan & $\begin{array}{c}\text { Ca: } 29 \mathrm{~A}, 36 \mathrm{~F}, 18 \mathrm{~N} \\
3 \mathrm{U} \\
\text { Co: } 62 \mathrm{~A}, 107 \mathrm{~F} \\
139 \mathrm{~N}, 15 \mathrm{U}\end{array}$ \\
\hline $\begin{array}{l}\text { Hwang et al., } \\
2014 \text { [77] }\end{array}$ & $74 / 85$ & NSCLC & Urine & LC-MS & N.A. & $\begin{array}{l}\text { Ca: } 64.0 \pm 10.3^{\mathrm{b}} \\
\text { Co: } 55.5 \pm 7.2^{\mathrm{b}}\end{array}$ & $\begin{array}{l}\text { Ca: } 45 \mathrm{M}, 29 \mathrm{~F} \\
\text { Co: } 23 \mathrm{M}, 62 \mathrm{~F}\end{array}$ & $\begin{array}{c}\text { Goyang, South } \\
\text { Korea }\end{array}$ & $\mathrm{N}$ \\
\hline Kim et al., 2015 [78] & $75 / 80$ & $\begin{array}{c}\text { AC (37) } \\
\text { SCC (30) } \\
\text { Other NSCLC (4) } \\
\text { Unknown (1) }\end{array}$ & $\begin{array}{l}\text { Fasting } \\
\text { plasma }\end{array}$ & $\begin{array}{l}\text { HPLC-electrospray } \\
\text { ionization mass } \\
\text { spectrometry }\end{array}$ & N.A. & $\begin{array}{l}\text { Ca: } 65.6 \pm 9.2^{b} \\
\text { Co: } 63.2 \pm 8.9^{b}\end{array}$ & $\begin{array}{l}\text { Ca: } 51 \mathrm{M}, 21 \mathrm{~F} \\
\text { Co: } 44 \mathrm{M}, 26 \mathrm{~F}\end{array}$ & South Korea & $\begin{array}{c}\text { Ca: } 40 \mathrm{~A}, 13 \mathrm{~F}, 19 \mathrm{~N} \\
\text { Co: } 8 \mathrm{~A}, 25 \mathrm{~F}, 34 \mathrm{~N}, \\
3 \mathrm{U}\end{array}$ \\
\hline $\begin{array}{l}\text { Klupczynska et al., } \\
\text { 2016a [79] }\end{array}$ & $90 / 62$ & $\begin{array}{l}\text { AC (40) } \\
\text { SCC (50) }\end{array}$ & Fasting serum & LC-MS/MS & N.A. & $\begin{array}{l}\text { Ca: } 64 \pm 6.9^{b} \\
\text { Co: } 62 \pm 8.8^{b}\end{array}$ & $\begin{array}{l}\text { Ca: } 58 \mathrm{M}, 32 \mathrm{~F} \\
\text { Co: } 40 \mathrm{M}, 22 \mathrm{~F}\end{array}$ & Poznan, Poland & $\begin{array}{l}\text { Ca: } 43 \mathrm{~A}, 46 \mathrm{~N}, 1 \mathrm{U} \\
\text { Co: } 11 \mathrm{~A}, 49 \mathrm{~N}, 3 \mathrm{U}\end{array}$ \\
\hline $\begin{array}{c}\text { Klupczynska et al., } \\
\text { 2016b [80] }\end{array}$ & $90 / 63$ & $\begin{array}{l}\text { AC (40) } \\
\text { SCC (50) }\end{array}$ & Fasting serum & LC-MS/MS & N.A. & $\begin{array}{l}\text { Ca: } 64 \pm 6.9^{b} \\
\text { Co: } 62 \pm 8.7^{b}\end{array}$ & $\begin{array}{l}\text { Ca: } 58 \mathrm{M}, 32 \mathrm{~F} \\
\text { Co: } 41 \mathrm{M}, 22 \mathrm{~F}\end{array}$ & Poznan, Poland & $\begin{array}{l}\text { Ca: } 43 \mathrm{~A}, 46 \mathrm{~N}, 1 \mathrm{U} \\
\text { Co: } 11 \mathrm{~A}, 49 \mathrm{~N}, 4 \mathrm{U}\end{array}$ \\
\hline Ni et al., 2016 [81] & $40 / 100$ & NR & Serum & LC-MS/MS & N.A. & $\begin{array}{l}\text { Ca: } 51-83^{\mathrm{d}} \\
\text { Co: } \mathrm{NR}\end{array}$ & $\begin{array}{l}\text { Ca: } 26 \mathrm{M}, 14 \mathrm{~F} \\
\text { Co: NR }\end{array}$ & Beijing, China & NR \\
\hline
\end{tabular}


Table 1. Cont.

\begin{tabular}{|c|c|c|c|c|c|c|c|c|c|}
\hline Reference, Year & $\begin{array}{l}\text { No. of Study } \\
\text { Participants } \\
\text { (Case/Control) }\end{array}$ & $\begin{array}{l}\text { Type of } \\
\text { Lung Cancer } \\
\text { Cases (n) }\end{array}$ & $\begin{array}{l}\text { Type of } \\
\text { Biological } \\
\text { Sample }\end{array}$ & $\begin{array}{l}\text { Method Used for } \\
\text { Metabolite } \\
\text { Identification }\end{array}$ & $\begin{array}{c}\text { Level of } \\
\text { Identification } \mathrm{g}\end{array}$ & $\begin{array}{l}\text { Age of Participants } \\
\text { at Recruitment }\end{array}$ & $\begin{array}{l}\text { Gender of } \\
\text { Participants }\end{array}$ & $\begin{array}{l}\text { Location of } \\
\text { Study (Study } \\
\text { Name, If } \\
\text { Applicable) }\end{array}$ & Smoking Status ${ }^{a}$ \\
\hline Yue et al., 2018 [31] & $20 / 20$ & SCLC & $\begin{array}{l}\text { Fasting } \\
\text { plasma }\end{array}$ & LC-MS/MS & $\begin{array}{l}\text { Levels 1, } 2 \text { (for } \\
\text { different } \\
\text { metabolites) }\end{array}$ & NR & NR & Beijing, China & NR \\
\hline $\begin{array}{l}\text { Kawamoto et al., } \\
2019 \text { [29] }\end{array}$ & $54 / 124$ & $\mathrm{AC}$ & Urine & Radioimmunoassay & N.A. & $\begin{array}{l}\text { Ca: } 66.6 \pm 10.0^{b} \\
\text { Co: } 44.2 \pm 12.9^{b}\end{array}$ & $\begin{array}{l}\text { Ca: } 23 \mathrm{M}, 31 \mathrm{~F} \\
\text { Co: } 52 \mathrm{M}, 72 \mathrm{~F}\end{array}$ & Tokyo, Japan & $\begin{array}{c}\text { Ca: } 30 \mathrm{~A} / \mathrm{F}, 24 \mathrm{~N} \\
\text { Co: } 124 \mathrm{~N}\end{array}$ \\
\hline $\begin{array}{l}\text { Klupczynska et al., } \\
2019 \text { [35] }\end{array}$ & $20 / 20$ & $\begin{array}{l}\text { AC (9) } \\
\text { SCC (11) }\end{array}$ & Fasting serum & $\begin{array}{c}\text { Triple quadrupole } \\
\text { tandem mass } \\
\text { spectrometer coupled } \\
\text { with HPLC }\end{array}$ & N.A. & $\begin{array}{l}\text { Ca: } 62 \pm 5^{b} \\
\text { Co: } 63 \pm 6^{b}\end{array}$ & $\begin{array}{l}\text { Ca: } 11 \mathrm{M}, 9 \mathrm{~F} \\
\text { Co: } 8 \mathrm{M}, 12 \mathrm{~F}\end{array}$ & Poznan, Poland & $\begin{array}{l}\text { Ca: } 12 \mathrm{~A}, 8 \mathrm{~F} / \mathrm{N} / \mathrm{U} \\
\text { Co: } 6 \mathrm{~A}, 14 \mathrm{~F} / \mathrm{N} / \mathrm{U}\end{array}$ \\
\hline Ni et al., 2019 [36] & $17 / 30$ & $\begin{array}{c}\text { AC (4) } \\
\text { SCC (5) } \\
\text { SCLC (5) } \\
\text { Other NSCLC (3) }\end{array}$ & Fasting serum & LC-MS/MS & N.A. & $\begin{array}{l}\text { Ca: } 53-77^{\mathrm{d}} \\
\text { Co: } 34-85^{\mathrm{d}}\end{array}$ & $\begin{array}{l}\text { Ca: } 13 \mathrm{M}, 4 \mathrm{~F} \\
\text { Co: } 23 \mathrm{M}, 7 \mathrm{~F}\end{array}$ & Beijing, China & $\begin{array}{c}\text { Ca: } 4 \mathrm{~A}, 5 \mathrm{~F}, 8 \mathrm{~N} \\
\text { Co: } 7 \mathrm{~A}, 6 \mathrm{~F}, 16 \mathrm{~N} \\
1 \mathrm{U}\end{array}$ \\
\hline $\begin{array}{l}\text { Pietzke et al., } \\
2019 \text { [25] }\end{array}$ & $56 / 50$ & $\begin{array}{l}\text { AC (31) } \\
\text { SCC (20) }\end{array}$ & $\begin{array}{l}\text { Fasting } \\
\text { plasma }\end{array}$ & GC-MS, LC-MS & N.A. & $\begin{array}{l}\text { Ca: } 66 \pm 9^{b} \\
\text { Co: } 48 \pm 14^{b}\end{array}$ & $\begin{array}{l}\text { Ca: 49M, 7F } \\
\text { Co: NR }\end{array}$ & NR & $\begin{array}{c}\text { Ca: } 28 \mathrm{~A}, 28 \mathrm{~F} / \mathrm{N} / \mathrm{U} \\
\text { Co: } N R\end{array}$ \\
\hline Zhang et al., 2019 [82] & $28 / 38$ & NR & $\begin{array}{l}\text { Fasting } \\
\text { plasma }\end{array}$ & HPLC-MS/MS & N.A. & $\begin{array}{l}\text { Ca: } 30-79^{\mathrm{d}} \\
\text { Co: } 20-79^{\mathrm{d}}\end{array}$ & $\begin{array}{l}\text { Ca: } 23 \mathrm{M}, 5 \mathrm{~F} \\
\text { Co: } 20 \mathrm{M}, 18 \mathrm{~F}\end{array}$ & Shenyang, China & $\begin{array}{c}\text { Ca: } 21 \mathrm{~A}, 7 \mathrm{~N} \\
\text { Co: } 15 \mathrm{~A}, 8 \mathrm{~N}, 15 \mathrm{U}\end{array}$ \\
\hline \multicolumn{10}{|c|}{ Studies reporting both the association between exposure to metabolite and lung cancer and the concentration of metabolites in lung cancer patients and controls $(\mathrm{n}=2)$} \\
\hline $\begin{array}{l}\text { Church et al., } \\
2009 \text { [83] }\end{array}$ & 100/100 & NR & $\begin{array}{l}\text { Non-fasting } \\
\text { serum }\end{array}$ & GC-MS & N.A. & $55-74^{\mathrm{d}}$ & $\begin{array}{l}\text { Ca: } 71 \mathrm{M}, 29 \mathrm{~F} \\
\text { Co: } 64 \mathrm{M}, 36 \mathrm{~F}\end{array}$ & $\begin{array}{c}\text { USA } \\
\text { (PLCO) }\end{array}$ & A \\
\hline $\begin{array}{l}\text { Skaaby et al., } \\
2014 \text { [84] }\end{array}$ & $\begin{array}{c}12204 \\
\text { (126 cases) }\end{array}$ & NR & Serum & $\begin{array}{l}\text { HPLC, immunoassay, } \\
\text { IDS-SYS 25-Hydroxy } \\
\text { Vitamin D method }\end{array}$ & N.A. & $18-71^{\mathrm{d}}$ & $5866 \mathrm{M}, 6338 \mathrm{~F}$ & $\begin{array}{c}\text { Denmark } \\
\text { (Monica10, } \\
\text { Inter99, } \\
\text { Health2006) }\end{array}$ & $\begin{array}{l}4554 \mathrm{~A}, 3401 \mathrm{~F} \\
\quad 4249 \mathrm{~N}\end{array}$ \\
\hline
\end{tabular}

AC Adenocarcinoma, ATBC Alpha-Tocopherol, Beta-Carotene Cancer Prevention, Ca Cases, CCHS Copenhagen City Heart Study, Co Controls, DCH Diet, Cancer and Health, DOM Diagnostisch Onderzoek (investigation) Mammacarcinoom, EC Epidermoid carcinoma, EPIC European Prospective Investigation into Cancer and Nutrition, ESTHER Epidemiologische Studie zu Chancen der Verhütung, Früherkennung und optimierten Therapie chronischer Erkrankungen in der älteren Bevölkerung, GC-MS/MS Gas chromatography-tandem mass spectrometry, KMCC Korean Multi-center Cancer Cohort, LC3 Lung Cancer Cohort Consortium, LCC Large cell carcinoma, LC-MS/MS Liquid chromatography-tandem mass spectrometry, MFhes Mini-Finland Health Survey, NMR Nuclear magnetic resonance, NR Not reported, NSCLC Non-small cell lung cancer, PC Pleomorphic carcinoma, PLCO Prostate, Lung, Colorectal, and Ovarian Cancer Screening Trial, PPP Pomeranian Pilot Lung Cancer Screening Programme, SCC Squamous cell carcinoma, SCCS Southern Community Cohort Study, SCHS Singapore Chinese Health Study, SCLC Small-cell lung cancer, SCS Shanghai Cohort Study, SWHS Shanghai Women's Health Study, UPLC-MS Ultra-high-performance liquid chromatography-tandem mass spectrometry, HPLC High-performance liquid chromatography, LC-MS/MS Liquid chromatography-tandem mass spectrometry, GC-MS Gas chromatography-mass spectrometry, GC-MS/MS Gas chromatography-tandem mass spectrometry, UPLC-ESI-QTOF-MS Ultra-performance liquid chromatography-electrospray-ionization-quadrupole time-of-flight mass spectrometry, N.A. not applicable. ${ }^{a}$ Abbreviations for smoking status are as follows: $A$, Active smoker; $E$, Ever smoker (active or former smoker); $F$, Former smoker; $N$, Never smoker; $P$, Passive smoker; $U$, Unknown smoking status. ${ }^{b}$ Data reported as mean $\pm \mathrm{SD} .{ }^{\mathrm{c}}$ Data reported as median and interquartile range. ${ }^{\mathrm{d}}$ Data reported as range (minimum-maximum). ${ }^{\mathrm{e}}$ Data reported as median, 5 th percentile and 95 th percentile. ${ }^{\mathrm{f}}$ Data reported as mean. ${ }^{\mathrm{g}}$ For untargeted metabolomics data: Level 1 identifies the compound by confirming with an authentic standard; Level 2 is matching to databases. 


\subsection{Quality of Eligible Studies}

The quality score of the included studies ranged from 3 to 9 (mean score $7.0 \pm 1.6$, Table 2). Most of the studies were of high quality $(\mathrm{n}=37,69.8 \%)$, while the remaining studies were mainly of moderate quality $(n=15,28.3 \%)$, with one being of low quality $(n=1,1.9 \%)$.

Table 2. Methodological quality assessment of included studies $(n=53)$ using a 9-point Newcastle-Ottawa Scale.

\begin{tabular}{|c|c|c|c|c|}
\hline Reference, Year & $\begin{array}{l}\text { Selection } \\
\text { (4) }{ }^{a}\end{array}$ & $\begin{array}{c}\text { Comparability } \\
\text { (2) }\end{array}$ & $\begin{array}{l}\text { Determination of } \\
\text { Exposure/Outcome } \\
\text { (3) }\end{array}$ & $\begin{array}{c}\text { Overall } \\
\text { Quality Score }\end{array}$ \\
\hline Kukreja et al., 1982 [39] & 1 & 2 & 3 & $6.8^{\mathrm{d}}$ \\
\hline Hendrick et al., 1988 [73] & 3 & 0 & 2 & 5 \\
\hline Preti et al., 1988 [34] & 3 & 0 & 2 & 5 \\
\hline de Waard et al., 1995 [50] & 4 & 1 & 2 & 7 \\
\hline Ellard et al., 1995 [51] & 4 & 2 & 2 & 8 \\
\hline London et al., 2000 [52] & 4 & 1 & 3 & 8 \\
\hline Proenza et al., 2003 [30] & 2 & 0 & 2 & 4 \\
\hline Masri et al., 2005 [40] & 3 & 2 & 3 & $9^{d}$ \\
\hline Boffetta et al., 2006 [53] & 4 & 2 & 3 & 9 \\
\hline Gencer et al., 2006 [74] & 2 & 0 & 2 & 4 \\
\hline Zhang et al., 2006 [75] & 1 & 0 & 2 & 3 \\
\hline Loft et al., 2007 [54] & 4 & 2 & 2 & 8 \\
\hline Esme et al., 2008 [76] & 3 & 1 & 2 & 6 \\
\hline Kilkkinen et al., 2008 [48] & 4 & 2 & 2 & 8 \\
\hline Church et al., 2009 [83] & 4 & 2 & 2 & 8 \\
\hline Hu et al., 2009 [26] & 3 & 0 & 2 & 5 \\
\hline Johansson et al., 2010 [55] & 3 & 2 & 3 & 8 \\
\hline Miyagi et al., 2011 [28] & 3 & 2 & 2 & 7 \\
\hline Timofeeva et al., 2011 [56] & 4 & 2 & 2 & 8 \\
\hline Weinstein et al., 2011 [57] & 4 & 2 & 2 & 8 \\
\hline Yuan et al., 2011 [58] & 4 & 2 & 3 & 9 \\
\hline Yuan et al., 2012 [59] & 4 & 2 & 3 & 9 \\
\hline Afzal et al., 2013 [49] & 4 & 2 & 3 & 9 \\
\hline Eom et al., 2013 [60] & 4 & 2 & 2 & 8 \\
\hline Kami et al., 2013 [33] & 2 & 2 & 3 & $7.9^{d}$ \\
\hline Okur et al., 2013 [41] & 3 & 2 & 3 & $9^{d}$ \\
\hline Shingyogi et al., 2013 [27] & 3 & 0 & 2 & 5 \\
\hline Chuang et al., 2014 [61] & 3 & 2 & 2 & 7 \\
\hline Hwang et al., 2014 [77] & 2 & 1 & 2 & 5 \\
\hline Mathe et al., 2014 [62] & 3 & 2 & 2 & 7 \\
\hline Skaaby et al., 2014 [84] & 3 & 2 & 3 & 8 \\
\hline Yuan et al., 2014 [63] & 4 & 2 & 3 & 9 \\
\hline Kim et al., 2015 [78] & 3 & 2 & 2 & 7 \\
\hline Wang et al., 2015 [64] & 3 & 2 & 2 & 7 \\
\hline Haznadar et al., 2016 [65] & 3 & 2 & 2 & 7 \\
\hline Klupczynska et al., 2016a [79] & 3 & 2 & 2 & 7 \\
\hline Klupczynska et al., 2016b [80] & 3 & 0 & 2 & 5 \\
\hline Ni et al., 2016 [81] & 3 & 2 & 2 & 7 \\
\hline $\begin{array}{l}\text { Ordóñez-Mena et al., } \\
\text { 2016-ESTHER [42] }\end{array}$ & 4 & 2 & 3 & 9 \\
\hline $\begin{array}{l}\text { Ordóñez-Mena et al., } \\
\text { 2016-TROMSØ [42] }\end{array}$ & 4 & 2 & 2 & 8 \\
\hline Yuan et al., 2016 [66] & 4 & 2 & 3 & 9 \\
\hline Yuan et al., 2017 [67] & 4 & 2 & 3 & 9 \\
\hline Fanidi et al., 2018 [68] & 3 & 2 & 2 & 7 \\
\hline Haznadar et al., 2018 [69] & 3 & 2 & 2 & 7 \\
\hline Larose et al., 2018 [70] & 2 & 2 & 2 & 6 \\
\hline
\end{tabular}


Table 2. Cont.

\begin{tabular}{ccccc}
\hline Reference, Year & $\begin{array}{c}\text { Selection } \\
\mathbf{( 4 )}^{\mathbf{a}}\end{array}$ & $\begin{array}{c}\text { Comparability } \\
\mathbf{( 2 )} \mathbf{b}^{\mathbf{b}}\end{array}$ & $\begin{array}{c}\text { Determination of } \\
\text { Exposure/Outcome } \\
\mathbf{( 3 )} \mathbf{c}^{\mathbf{c}}\end{array}$ & $\begin{array}{c}\text { Overall } \\
\text { Quality Score }\end{array}$ \\
\hline Yue et al., 2018 [31] & 3 & 1 & 2 & 6 \\
Gao et al., 2019a-ESTHER [43] & 3 & 2 & 3 & 8 \\
Gao et al., 2019a-TROMSØ [43] & 3 & 2 & 2 & 7 \\
Gao et al., 2019b [71] & 3 & 2 & 3 & 7 \\
Kawamoto et al., 2019 [29] & 3 & 2 & 2 & 6 \\
Klupczynska et al., 2019 [35] & 2 & 2 & 2 & 7 \\
Ni et al., 2019 [36] & 3 & 2 & 2 & 4 \\
Pietzke et al., 2019 [25] & 2 & 0 & 2 & 8 \\
Seow et al., 2019 [72] & 4 & 2 & 2 & 4 \\
Zhang et al., 2019 [82] & 2 & 0 & 2 & 7 \\
\hline
\end{tabular}

ESTHER Epidemiologische Studie zu Chancen der Verhütung, Früherkennung und optimierten Therapie chronischer Erkrankungen in der älteren Bevölkerung cohort study, TROMSØ Tromsø study. ${ }^{a}$ A maximum of four points may be awarded to the study based on: (for case-control studies) adequacy of case definition, representativeness of cases, selection of controls, and definition of controls; or, (for cohort studies) representativeness of exposed cohort, selection of non-exposed cohort, ascertainment of exposure, and demonstration that outcome of interest was not present at start of study. ${ }^{b}$ A maximum of two points may be awarded to the study, based on the comparability between cases and controls (for case-control studies) or comparability between the exposed and non-exposed group (for cohort studies). One point was awarded if the study adjusted for age, and the other point was awarded if the following factors were controlled for or stratified during analysis: age, smoking status, gender. ${ }^{c}$ A maximum of three points may be awarded to the study, based on: (for case-control studies) ascertainment of exposure, same method of ascertainment for cases and controls, and same non-response rate or, (for cohort studies) assessment of outcome, duration of follow-up for outcome, and adequacy of follow-up of cohorts. ${ }^{\mathrm{d}}$ For self-controlled case-control studies, a modified version of the NOS was used, with the exclusion of the question on the definition of controls, such that these studies could have a maximum of 8 points (instead of 9 points). The overall quality score of each of these studies was scaled to a maximum score of 9 points, in order to facilitate comparison across all studies.

\subsection{Association between Metabolites and Lung Cancer Risk}

A total of 43 individual metabolites and 10 groups of metabolites were reported across all the identified categorical studies (Table S2). Of these, 18 meta-analyses were performed on 12 metabolites, which were: 2-hydroxyethyl mercapturic acid (HEMA), 3-hydroxypropyl mercapturic acid (HPMA), 4-hydroxybut-2-yl mercapturic acid (HBMA), cotinine, creatine riboside, cortisol sulfate, folate, methionine, $\mathrm{N}$-acetylneuraminic acid (NANA), pyridoxal 5'-phosphate, r-1,t-2,3,c-4-tetrahydroxy-1,2,3,4-tetrahydrophenanthrene (PheT) and S-phenyl mercapturic acid (SPMA); and five groups of metabolites, which were:

- Total 3-hydroxycotinine (3-HC) (defined as the sum of concentrations of 3-HC and its glucuronide),

- Total cotinine (defined as the sum of concentrations of cotinine and its glucuronide),

- Total nicotine (defined as the sum of concentrations of nicotine and its glucuronide),

- Total NNAL (defined as the sum of concentrations of 4-(methylnitrosamino)-1-(3-pyridyl)1-butanol (NNAL) and its glucuronides), and,

- Total nicotine equivalent (TNE) (defined as the sum of the concentration of nicotine, cotinine, 3-HC and their respective glucuronides).

Twelve meta-analyses were performed using the fixed-effects model, while the other six were performed using the random-effects model. Meta-analyses were not performed on the remaining metabolites as they were solely reported in individual studies (Table S3).

Of the serum/plasma metabolites that were meta-analyzed, an increased concentration of cotinine (OR $=14.19,95 \% \mathrm{CI}=2.92$ to 69.00 , Table 3 and Figure S1a) and decreased concentration of folate $(\mathrm{OR}=0.82,95 \% \mathrm{CI}=0.72$ to 0.94 , Table 3 and Figure S1b) were significantly associated with increased lung cancer risk. 
Table 3. Odds ratio (OR) and $95 \%$ confidence interval (CI) of the association of lung cancer in relation to metabolites that achieved statistical significance. Refer to Figure S1 for the forest plot of each metabolite.

\begin{tabular}{|c|c|c|c|c|c|c|}
\hline Metabolite. & OR & $95 \% \mathrm{CI}$ & $\begin{array}{l}\text { No. of } \\
\text { Studies }\end{array}$ & $I^{2}(\%)$ & $\begin{array}{c}\text { Cochran's Q } \\
\text { Test's } p \text {-Value }\end{array}$ & Forest Plot \\
\hline \multicolumn{7}{|c|}{ Serum/Plasma } \\
\hline Folate & 0.82 & $0.72-0.94$ & 2 & 47.7 & 0.167 & Figure S1b \\
\hline \multicolumn{7}{|c|}{ Urine } \\
\hline Creatine Riboside $^{+}$ & 3.30 & $1.33-8.15$ & 2 & 84.7 & 0.011 & Figure S1c \\
\hline PheT & 2.49 & $1.53-4.05$ & 2 & 0.0 & 0.673 & Figure S1e \\
\hline Total 3-HC (3-HC + 3-HC-Gluc) & 3.71 & $2.41-5.72$ & 2 & 0.0 & 0.499 & Figure S1f \\
\hline Total Cotinine (Cotinine + Cotinine-Gluc) & 3.53 & $2.62-4.77$ & 3 & 0.0 & 0.406 & Figure S1g \\
\hline Total Nicotine (Nicotine + Nicotine-Gluc) & 2.51 & $1.71-3.70$ & 2 & 8.9 & 0.295 & Figure S1h \\
\hline Total NNAL (NNAL + NNAL-Glucs) & 2.17 & $1.63-2.89$ & 3 & 28.3 & 0.248 & Figure S1i \\
\hline $\begin{array}{l}\text { Total Nicotine Equivalent (Total nicotine }+ \\
\text { Total cotinine }+ \text { Total 3-HC) }\end{array}$ & 3.75 & $2.45-5.73$ & 2 & 16.3 & 0.274 & Figure S1j \\
\hline
\end{tabular}

${ }^{\dagger}$ Random-effects models were used for this metabolite.

Among urinary metabolites that were analyzed, increased concentration of creatine riboside $(\mathrm{OR}=3.30,95 \% \mathrm{CI}=1.33$ to 8.15 , Table 3 and Figure S1c), NANA (OR $=2.01,95 \% \mathrm{CI}=1.49$ to 2.72 , Table 3 and Figure S1d), PheT (OR $=2.49,95 \% \mathrm{CI}=1.53$ to 4.05, Table 3 and Figure S1e), total 3-HC $(\mathrm{OR}=3.71,95 \% \mathrm{CI}=2.41$ to 5.72 , Table 3 and Figure $\mathrm{S} 1 \mathrm{f})$, total cotinine $(\mathrm{OR}=3.53,95 \% \mathrm{CI}=2.62$ to 4.77, Table 3 and Figure S1g), total nicotine ( $\mathrm{OR}=2.51,95 \% \mathrm{CI}=1.71$ to 3.70, Table 3 and Figure S1h), total NNAL ( OR $=2.17,95 \% \mathrm{CI}=1.63$ to 2.89 , Table 3 and Figure S1i) and $\mathrm{TNE}(\mathrm{OR}=3.75,95 \% \mathrm{CI}=2.45$ to 5.73, Table 3 and Figure S1j) were significantly associated with increased lung cancer risk.

No statistically significant associations between the exposure of eight metabolites (plasma/serum pyridoxal 5'-phosphate and methionine, and urinary cortisol sulfate, cotinine, HBMA, HEMA, HPMA and SPMA) and lung cancer risk were observed (Figure S3).

Results were stratified by gender for cotinine, pyridoxal 5'-phosphate, total 3-HC, total cotinine, total nicotine, total NNAL and TNE (Figures S1f-j and S4a-c), and by smoking status for cotinine, folate, methionine and pyridoxal 5'-phosphate (Figure S4d-h). No clear trend between men and women were observed with respect to the association between metabolites and lung cancer risk. Among the stratified results, active or former smokers have a stronger association between cotinine, folate and pyridoxal 5'-phosphate exposure and lung cancer risk, when compared to passive or never smokers.

Cotinine exposure was not significantly associated with lung cancer risk among never smokers (serum/plasma cotinine: $\mathrm{OR}=1.06,95 \% \mathrm{CI}=0.52$ to 2.14 , Figure S4d) and passive smokers (urinary cotinine: $\mathrm{OR}=2.40,95 \% \mathrm{CI}=0.70$ to 8.30 , Figure $\mathrm{S} 4 \mathrm{~h}$ ), although it remained significant for active smokers (serum/plasma cotinine: $\mathrm{OR}=4.15,95 \% \mathrm{CI}=2.59$ to 6.66 , Figure S4d; urinary cotinine: $\mathrm{OR}=9.80,95 \% \mathrm{CI}=4.50$ to 21.30 , Figure $\mathrm{S} 4 \mathrm{~h}$ ). The inverse association between serum/plasma folate levels and lung cancer risk was stronger among active smokers $(\mathrm{OR}=0.75,95 \% \mathrm{CI}=0.42$ to 1.32) and former smokers ( $\mathrm{OR}=0.64,95 \% \mathrm{CI}=0.51$ to 0.80 ) when compared with never smokers $(\mathrm{OR}=0.86,95 \% \mathrm{CI}=0.65$ to 1.13 ) (Figure S4e). The inverse association between serum/plasma pyridoxal $5^{\prime}$-phosphate and lung cancer risk was statistically significant among active smokers $(\mathrm{OR}=0.77,95 \% \mathrm{CI}=0.65$ to 0.92$)$ and former smokers $(\mathrm{OR}=0.63,95 \% \mathrm{CI}=0.45$ to 0.89$)$, but not among never smokers $(\mathrm{OR}=0.78,95 \% \mathrm{CI}=0.20$ to 3.09) (Figure $\mathrm{S} 4 \mathrm{~g}$ ).

\subsection{Quantitative Difference in Metabolite Level between Lung Cancer Patients and Controls}

A total of 255 metabolites were reported across all the identified concentration studies (Table S4). Of these, 40 meta-analyses were performed on 21 types of amino acid in plasma, 11 types of amino acid in serum and eight types of carnitines in serum. Two meta-analyses were performed using the fixed-effects model, while the other 38 were performed using the random-effects model. The remaining metabolites were reported in only individual studies and thus were not meta-analyzed (Table S5). 
Of the meta-analyzed metabolites, lower concentration of plasma methionine (WMD $=-2.04,95 \%$ $\mathrm{CI}=-4.01$ to -0.06 , Table 4 and Figure S2a) and tryptophan (WMD $=-6.85,95 \% \mathrm{CI}=-11.07$ to -2.63 , Table 4 and Figure S2b) and higher concentration of plasma proline (WMD = 15.98, 95\% CI =6.59 to 25.37, Table 4 and Figure S2c) were found in lung cancer patients, compared with healthy controls. The differences in concentration of other amino acids and carnitines between lung cancer patients and controls were not statistically significant (Figure S5).

Table 4. Weighted mean difference (WMD) and 95\% confidence interval (CI) of the of the plasma metabolite concentration between lung cancer patients and healthy controls, for metabolites that achieved statistical significance. Refer to Figure S2 for the forest plot of each metabolite.

\begin{tabular}{|c|c|c|c|c|c|c|}
\hline Metabolite & $\begin{array}{c}\text { WMD } \\
(\mu \mathrm{mol} / \mathrm{L})\end{array}$ & $\begin{array}{c}95 \% \text { CI } \\
(\mu \mathrm{mol} / \mathrm{L})\end{array}$ & $\begin{array}{l}\text { No. of } \\
\text { Studies }\end{array}$ & $I^{2}(\%)$ & $\begin{array}{c}\text { Cochran's Q } \\
\text { Test's } p \text {-Value }\end{array}$ & Forest Plot \\
\hline \multicolumn{7}{|c|}{ Plasma } \\
\hline Methionine $^{+}$ & -2.04 & $-4.01--0.06$ & 5 & 86.0 & $<0.001$ & Figure S2a \\
\hline Tryptophan ${ }^{\dagger}$ & -6.85 & $-11.07--2.63$ & 4 & 87.1 & $<0.001$ & Figure S2b \\
\hline Proline $^{+}$ & 15.98 & 6.59-25.37 & 6 & 83.6 & $<0.001$ & Figure S2c \\
\hline
\end{tabular}

\section{Discussion}

\subsection{Overview}

This systematic literature review and meta-analysis aimed to provide a review of the current understanding of the association between metabolites and lung cancer risk. We had included 53 studies, involving 297 metabolites across six different types of biological samples. Although many metabolomics studies on lung cancer were included in this review, the data on any given metabolite is limited, due to the differences in the research focus of each study. Thus, this discussion will primarily focus on the metabolites that were meta-analyzed.

Meta-analyses were performed on 45 metabolites. Our findings indicated that three amino acids (methionine, tryptophan and proline), two smoking-related metabolites (cotinine and PheT) and five groups of smoking-related metabolites (total 3-HC, total cotinine, total nicotine, total NNAL and TNE), a vitamin (folate), a sialic acid (NANA), and a novel compound (creatine riboside) had statistically significant association with lung cancer risk.

\subsection{Amino Acids}

Amino acids have been reported to be associated with other types of cancer in earlier reviews [88-91]. The amino acids identified in our meta-analyses (methionine, tryptophan and proline) were identified in previous reviews, although the direction of association is different for proline (i.e., positive association in our meta-analysis on lung cancer but inverse association for other cancer types) $[88,90,91]$. A possible explanation for the discrepancy is that different cancer types have dissimilar metabolism, therefore the association between the patient's host protein metabolism and amino acid levels with cancer risk may differ between cancer types [92-95].

\subsubsection{Methionine}

Results from concentration studies (Figure S2a) suggested that plasma methionine concentration was lower in lung cancer patients. The findings from Kami et al. [33] also identified that the concentration of methionine was increased in tumor tissue of lung cancer patients (Table S5). These observations are largely consistent with the current understanding of the roles that methionine plays in cancer cell proliferation.

The influential roles of methionine in cancer cell growth has been well-documented [96-99]. Methionine is involved in several critical activities in cancer cells, such as nucleotide biosynthesis 
via the one-carbon metabolism pathway [100-102] and protein synthesis [103]. Methionine is also a precursor metabolite of S-adenosylmethionine, a co-substrate involved in methyl group donation, for cellular processes such as epigenetic control and protein methylation [100,104,105]. Previous reviews have identified that the DNA methylation patterns in lung cancer were altered, where genome-wide hypomethylation, and hypermethylation of the promoter regions of several tumor suppressor genes, such as DAPK, RASSF1A and RAR $\beta$, were commonly observed [106,107]. It is thus unsurprising to observe an increased demand for methionine in cancer cells, given the multi-faceted role it plays in oncogenesis. Consequently, cancer cells increase its uptake of essential amino acids, such as methionine, through the up-regulation of essential amino acid transporters [108-110].

Methionine is also involved in glutathione formation, which serves as an antioxidant [111]. Lowered glutathione level is a biomarker of oxidative stress [112], and may contribute to chronic inflammation and cancer development [113]. These observations suggest that lowered methionine levels may also play a role in the precipitation of cancer cell proliferation, through the reduction in antioxidant capacity. Methionine is also a direct target of reactive oxygen species (ROS) and acts as a ROS scavenger [114,115].

\subsubsection{Tryptophan}

Similar to methionine, our findings indicated that the plasma levels of tryptophan were lowered in lung cancer patients (Figure S2b). Furthermore, findings from Chuang et al. [61] suggested that increased kynurenine and 3-hydroxykynurenine levels were associated with lung cancer risk, although the association was not statistically significant (Table S3). These observations could be explained by a potential mechanism that cancer cells use to bypass detection by the host immune system [116].

Tryptophan is a precursor molecule in the kynurenine pathway [117], which synthesizes several metabolites with immunosuppressive activity. The metabolites then suppresses T-cell proliferation and alters NK cell function [118]. A review by Heng et al. [119] identified that increased expression of indoleamine-2,3-dioxygenase 1 (IDO1), an enzyme involved in the synthesis of kynurenine from tryptophan, is positively correlated with poorer cancer prognosis across different cancers, including lung cancer. They further illustrated that an increase in IDO1 would up-regulate the production of kynurenine and its metabolites, which were then used to suppress T-cell activity.

\subsubsection{Proline}

Our findings indicated that the plasma levels of proline were higher among lung cancer patients (Figure S2c). Unlike methionine and tryptophan, the biochemical rationale behind the increase in plasma concentrations of proline is unclear.

The role of proline in lung carcinogenesis is an area of active research. A recent review by Phang et al. provided an abridged overview of the functionality of proline in cancer cells, as a source for cellular energy production and as an intermediate between the urea cycle and Krebs cycle [120]. Several studies have identified that overexpression of proline dehydrogenase, the enzyme involved in proline degradation, promotes cancer progression [121-123]. Future studies could explore the relationship between increased circulating proline concentrations and lung cancer risk.

\subsection{Folate}

Folate is a metabolite in the folate cycle, which is part of the one-carbon metabolism pathway [100-102]. Folate is coupled with the methionine cycle as one of its metabolites, 5-methyltetrahydrofolate, is involved in the regeneration of methionine from homocysteine [100-102]. Similar to methionine, folate is involved in nucleotide biosynthesis. 10-formyltetrahydrofolate, a metabolite in the folate cycle, serves as a formyl group donor for the formation of carbon- 2 and carbon-8 of the purine ring in de novo purine synthesis [100-102].

Our stratified analysis has identified that active and former smokers have a stronger inverse association between folate levels and lung cancer risk when compared with never smokers (Figure S4e). 
A possible explanation for this observation may be the confounding effect of exposure to tobacco smoke. The authors of the respective studies cautioned that despite adjusting for the cotinine level, the results may still be confounded by smoking status, considering the influence that smoking has on lung cancer risk $[55,68]$.

\subsection{Smoking-Related Metabolites}

Smoking is well-established as a significant risk factor for lung cancer [124-127]. More than 5000 compounds were identified in tobacco smoke, among which, over 70 compounds were identified as carcinogenic [128-130]. In this study, we conducted meta-analyses on nine smoking-related metabolites, namely nicotine, cotinine, 3-HC, PheT, NNAL, HBMA, HEMA, HPMA and SPMA, either as single metabolite, or as metabolite groups (as are the cases for the meta-analyses on total nicotine, total cotinine, total 3-HC, total NNAL and TNE).

\subsubsection{Nicotine and Cotinine}

Cotinine is a major metabolite from nicotine metabolism in humans, accounting for $70-80 \%$ of the metabolites formed [131]. Cotinine then undergo further metabolism before being excreted [132], with 3-HC being a main metabolite found in urine [133]. Although nicotine and cotinine are associated with lung cancer risk, it is imperative to note that neither are carcinogens. Several studies have shown that nicotine and cotinine neither induced nor influenced lung tumorigenesis [134,135].

Results from our meta-analyses suggested that increased serum/plasma cotinine, urinary total 3-HC, cotinine and nicotine exposure were significantly associated with higher lung cancer risk (Figure S1a,f-h). Urinary cotinine was also associated with higher lung cancer risk, albeit not statistically significant (Figure S3d). These observed associations were noteworthy as nicotine exposure is a consequence of cigarette smoke exposure, which itself contains carcinogens that may induce tumorigenesis [128-130].

From the stratified analysis by smoking status, cotinine exposure was associated with lung cancer risk among active smokers, but not among passive or never smokers (Figure S4d,h). Our meta-analyses have also identified that urinary TNE, a biomarker that demonstrated high correlation with smoking [136,137], is positively associated with lung cancer risk (Figure S1j). These results further exemplified the significance of smoking, rather than nicotine exposure, as a risk factor for lung cancer.

\subsubsection{PheT}

Polycyclic aromatic hydrocarbons (PAHs), are a class of compounds that are well-established as carcinogens [138], with well-documented association between PAH exposure and lung cancer risk $[139,140]$. Of these, benzoapyrene $(\mathrm{BaP})$, a compound known for its carcinogenic effects, was frequently studied and often used as a reference compound for the evaluation of carcinogenicity of other PAHs [141]. The International Agency for Research on Cancer (IARC) classified several PAHs as carcinogens or potential carcinogens [142]. PAHs are pro-carcinogens [143-146], with its carcinogenicity potentiated by multi-step biotransformation of the parent compounds, through several metabolic pathways, such as CYP1A1/1B1 and epoxide hydrolase pathway, and aldo-keto reductases pathway [138]. Particularly, PAHs were activated through the formation of bay-region diol epoxides [147]. The carcinogenic metabolites then form DNA adducts, causing errors in DNA replication and altered epigenetic controls, and may contribute to carcinogenesis [143-146].

PheT, a metabolite of non-carcinogenic phenanthrene, is an established surrogate measure for carcinogenic PAH exposure [147]. Results from our meta-analysis showed that increased PheT exposure was associated with higher lung cancer risk (Figure S1e), consistent with the current understanding of the roles that PAH may play in lung cancer. 


\subsubsection{NNAL}

NNAL is a metabolite of 4-(N-Nitrosomethylamino)-1-(3-pyridyl)-1-butanone (NNK), one of the compounds found in cigarette smoke [148]. NNAL can be further metabolized through glucuronidation into either the $\mathrm{O}$ or N glucuronides [149]. Both NNK and NNAL are pro-carcinogens [149]. On its own, both compounds do not have any carcinogenic effect [149]. However, its metabolites (such as $\alpha$-methylenehydroxy-NNK and 4-(3-pyridyl)-4-oxobutane-1-diazohydroxide) are carcinogenic and may cause DNA damage through the formation of DNA adducts [149].

Total NNAL, the sum of urinary NNAL and its glucuronides, were validated biomarkers for NNK exposure [150]. Our meta-analyses suggested that increased urinary total NNAL was significantly associated with higher lung cancer risk (Figure S1i), further substantiating the current theory of the role of NNAL (and NNK) in carcinogenesis.

\subsubsection{HBMA, HEMA, HPMA and SPMA}

HBMA, HEMA, HPMA and SPMA are mercapturic acid metabolites of crotonaldehyde, ethylene oxide, acrolein and benzene respectively, and are validated biomarkers for the exposure to the respective parent compounds [151]. Our findings suggested that increased exposure to any of these four metabolites were not associated with lung cancer risk (Figure S3e-h).

Crotonaldehyde is a volatile $\alpha, \beta$-unsaturated carbonyl compound [152]. Although crotonaldehyde was shown to induce liver tumors in rats [153], to date, insufficient evidence was found to show that crotonaldehyde exposure is associated with lung cancer risk. According to the IARC, crotonaldehyde is "not classifiable as to its carcinogenicity to humans" [154].

Ethylene oxide is a volatile cyclic ether [155]. The IARC classified ethylene oxide as "carcinogenic to humans", based on animal data [154]. However, an earlier review suggested that current evidence was insufficient to conclude the carcinogenicity of ethylene oxide on human. Similarly, our meta-analysis did not find any association between ethylene oxide exposure and lung cancer risk $(\mathrm{OR}=1.01$, $95 \% \mathrm{CI}=0.64$ to 1.59 ; Figure $\mathrm{S3f}$ ).

Acrolein is a volatile $\alpha, \beta$-unsaturated aldehyde [156]. Although acrolein had demonstrated carcinogenicity in in vitro models [157], there is insufficient evidence to show that acrolein exposure is associated with lung cancer risk to date. According to the IARC, acrolein is "not classifiable as to its carcinogenicity to humans" [154].

Benzene is a volatile aromatic compound [155]. An earlier review summarized the carcinogenic effects of benzene for different tumors in animals [158]. A review by IARC summarized that benzene exposure was associated with increased lung cancer risk in a few studies, although most studies have shown no association [159]. Similarly, our study suggested that increased exposure to SPMA is associated to higher lung cancer risk, albeit not statistically significant $(\mathrm{OR}=1.28,95 \% \mathrm{CI}=0.83$ to 1.96; Figure S3h). The IARC classified benzene as "carcinogenic to humans", due to its association with several types of leukemia and lymphoma [154,159].

\subsection{NANA}

Increased concentrations of NANA have been associated with several cancer types [160-165]. NANA is used in the formation of glycans [166], which in turn play several influential roles in cells, such as inducing proper folding of newly synthesized proteins [167], cell signaling and adhesion [168].

Anomalous glycosylation is increasingly recognized as a hallmark of cancer [169]. Tumor cells are known to produce increased amounts of glycans on the plasma membrane [170]. Excessive glycosylation increases the negative charge on plasma membrane of tumor cells, promoting cell detachment and encouraging metastasis [171].

Results from our meta-analysis concluded that increased NANA exposure was associated with higher lung cancer risk (Figure S1d). Notably, the observation of increased NANA may be due to increased turnover and shedding of cancer cells, resulting in glycans being released into the serum [166]. 


\subsection{Creatine Riboside}

Creatine riboside is a novel metabolite that was not previously identified in other cancer studies. To date, no studies were performed to elucidate the potential mechanisms behind the elevation of creatine riboside concentration in lung cancer patients. In the study that identified creatine riboside as a statistically significant metabolite, Mathe et al. [62] hypothesized that higher concentrations of creatine riboside may be the consequence of both increased creatine concentrations and higher phosphate turnover within tumor cells.

\subsection{Strengths and Limitations of This Study}

Previous reviews summarized recent work regarding the approaches in the identification of metabolites that could serve as biomarkers for lung cancer and enumerated several potential biomarkers [22,23]. On the other hand, previous meta-analyses focused only on exposure to vitamin $\mathrm{D}[172,173]$. Our current study further expanded on the existing reviews and meta-analyses by identifying other types of metabolites and quantifying either the differences in concentrations between lung cancer patients and controls or the lung cancer risk associated with levels of metabolite. By not restricting the type of metabolite in our inclusion criteria, we included metabolites across different metabolism pathways to provide a comprehensive overview of the current progress in the field.

The results of our meta-analysis should be interpreted with the following caveats in mind. Firstly, most of the metabolites identified were only reported as individual, small-scale studies. The limited quantity of evidence found for most metabolites suggests that further studies are merited in order to validate the feasibility of using these metabolites as biomarkers for lung cancer. Furthermore, about one-third ( $n=16,30.2 \%$ ) of the included studies were of low-to-moderate quality. Thus, with these points in consideration, the current results should be interpreted with prudence, and future well-designed, large-scale studies are warranted.

Secondly, we could not perform extensive stratified analyses to explore sources of clinical heterogeneity. Due to the limited studies available on each metabolite analyzed, we were only able to perform subgroup analysis by gender and smoking status for those metabolites that were investigated by sufficient number of studies. In addition, most studies did not include the definitions for smoking status, nor did they provide stratified results by smoking status. Thus, we could not account for any possible differences in smoking status. Some of the included studies only presented the combined results of plasma and serum, therefore we were unable to separate them in our analysis [56,68,70]. Furthermore, we could not perform stratified analyses by histological subtype, stage of lung cancer and study design. Although the majority of studies reported the histological subtype of lung cancer patients (Table 1), most studies did not report stratified results based on lung cancer subtypes. Moreover, only a handful of studies reported the lung cancer stage. Several reviews on other cancers, such as colorectal cancer and gastric cancer, have identified that the metabolome profile is different between early-stage and late-stage cancer [88,90]. Thus, future studies would benefit by exploring the influence of lung cancer stage on the evaluated associations in relation to different metabolites.

We noted some heterogeneity in the identified studies. The heterogeneity may be attributed to factors such as ethnicity and smoking status. We recognize that this is a knowledge gap in the current state of research, and more studies should be performed to facilitate the identification of possible factors for heterogeneity. The method used for metabolite identification may also be a possible source of heterogeneity. Considering that studies used different analytical platforms and methodology to identify and quantify the metabolites (Table 1), more work should be done to further harmonize the workflow adopted by researchers in metabolite quantification. We recommend researchers to follow the best practices for the quantification of the metabolites, such as that proposed by Lu et al. [174], and adopt minimum reporting standards, such as that proposed by the Metabolomics Standards Initiative [175], to facilitate the replication of studies and allowing for more meaningful comparisons between different studies. 
Thirdly, we were unable to ascertain if the patients in the identified studies were free of co-morbidities, or if it was adjusted for. Several co-morbidities, such as chronic obstructive pulmonary disease (COPD), diabetes mellitus (DM) and other malignancies, were identified to be more prevalent in lung cancer patients compared to the general population [176-178]. The metabolomic profile of patients in disease states, such as COPD [179] and DM [180], were identified to be different from that of a normal population. While we excluded studies involving patients with other malignancies or having a history of any malignancy, we could not ascertain if patients were free of co-morbidities at the time of sample collection. It is beneficial for future studies to consider the participants' co-morbidities as a criterion for recruitment, or to report it as part of the baseline characteristics of their participants.

\section{Conclusions}

This review identified several metabolites that are significantly associated with lung cancer. Amino acids, smoking-related metabolites, folate, NANA and creatine riboside warrant further investigation for use as potential biomarkers. Although a substantial number of studies were reviewed, meta-analyses could only be performed on a subset of the identified metabolites, as most metabolites were solely studied in individual studies. Out of the analyzed metabolites, plasma samples of amino acids may hold more promise. Further studies are warranted to elucidate the possible link between amino acid levels and lung cancer, and to validate the use of these metabolites as potential biomarkers of lung cancer in a larger population.

Supplementary Materials: The following are available online at http://www.mdpi.com/2218-1989/10/9/362/s1, List S1: List of abbreviations used, List S2: Preferred Reporting items for Systematic Reviews and Meta-analysis (PRISMA) checklist, Table S1: Literature search strategy used for electronic databases, Table S2: Metabolites reported in categorical studies, Table S3: Metabolites solely reported in individual categorical studies, Table S4: Metabolites reported in concentration studies, Table S5: Metabolites solely reported in individual concentration studies, Figure S1: Effect size (ES; i.e., odds ratio (OR)) and 95\% confidence interval (CI) of the association of lung cancer in relation to metabolites that achieved statistical significance, Figure S2: Weighted mean difference (WMD) and $95 \%$ confidence interval (CI) of the plasma metabolite concentration between lung cancer patients and healthy controls using a random-effects model, for metabolites that achieved statistical significance, Figure S3: Effect size (ES; i.e., odds ratio (OR)) and 95\% confidence interval (CI) of the association of lung cancer in relation to levels of metabolite, for metabolites that did not achieve statistical significance, Figure S4: Effect size (ES; i.e., odds ratio (OR)) and 95\% confidence interval (CI) of the association of lung cancer in relation to levels of metabolite, stratified by gender (for a-c) and smoking status (for d-h), Figure S5: Weighted mean difference (WMD) and 95\% confidence interval (CI) of the metabolite concentration between lung cancer patients and healthy controls for metabolites that did not achieve statistical significance.

Author Contributions: Conceptualization, W.J.S.; methodology, K.B.L, W.-P.Y. and W.J.S.; data analysis, K.B.L. and L.A.; writing —original draft preparation, K.B.L; writing—review and editing, K.B.L, W.-P.Y. and W.J.S.; supervision, W.-P.Y. and W.J.S.; funding acquisition, W.J.S. All authors have read and agreed to the published version of the manuscript.

Funding: The APC was funded by NUS Start-up Grant.

Conflicts of Interest: The authors declare no conflict of interest.

\section{References}

1. International Agency for Research on Cancer, WHO. Latest Global Cancer Data: Cancer Burden Rises to 18.1 Million New Cases and 9.6 Million Cancer Deaths in 2018; International Agency for Research on Cancer, World Health Organization: Geneva, Switzerland, 2018.

2. Travis, W.D.; Brambilla, E.; Burke, A.P.; Marx, A.; Nicholson, A.G. Introduction to The 2015 World Health Organization Classification of Tumors of the Lung, Pleura, Thymus, and Heart. J. Thorac. Oncol. 2015, 10, 1240-1242. [CrossRef]

3. Youlden, D.R.; Cramb, S.M.; Baade, P.D. The International Epidemiology of Lung Cancer: Geographical Distribution and Secular Trends. J. Thorac. Oncol. 2008, 3, 819-831. [CrossRef] [PubMed]

4. Wong, M.C.S.; Lao, X.Q.; Ho, K.-F.; Goggins, W.B.; Tse, S.L.A. Incidence and mortality of lung cancer: Global trends and association with socioeconomic status. Sci. Rep. 2017, 7, 14300. [CrossRef] [PubMed] 
5. World Cancer Research Fund/American Institute for Cancer Research. Diet, Nutrition, Physical Activity and Lung Cancer; World Cancer Research Fund/American Institute for Cancer Research: Islington, London, UK, 2018.

6. Torre, L.A.; Siegel, R.L.; Ward, E.M.; Jemal, A. Global Cancer Incidence and Mortality Rates and Trends-An Update. Cancer Epidemiol. Biomark. Prev. 2016, 25, 16-27. [CrossRef] [PubMed]

7. Lam, W.K.; White, N.W.; Chan-Yeung, M.M. Lung cancer epidemiology and risk factors in Asia and Africa. Int. J. Tuberc. Lung Dis. 2004, 8, 1045-1057. [PubMed]

8. American Lung Association. Lung Cancer Fact Sheet. Available online: https://www.lung.org/lung-healthand-diseases/lung-disease-lookup/lung-cancer/resource-library/lung-cancer-fact-sheet.html (accessed on 1 April 2020).

9. Blandin Knight, S.; Crosbie, P.A.; Balata, H.; Chudziak, J.; Hussell, T.; Dive, C. Progress and prospects of early detection in lung cancer. Open Biol. 2017, 7, 170070. [CrossRef]

10. Walters, S.; Maringe, C.; Coleman, M.P.; Peake, M.D.; Butler, J.; Young, N.; Bergstrom, S.; Hanna, L.; Jakobsen, E.; Kolbeck, K.; et al. Lung cancer survival and stage at diagnosis in Australia, Canada, Denmark, Norway, Sweden and the UK: A population-based study, 2004-2007. Thorax 2013, 68, 551-564. [CrossRef]

11. Clish, C.B. Metabolomics: An emerging but powerful tool for precision medicine. Mol. Case Stud. 2015, 1, a000588. [CrossRef]

12. Wishart, D.S. Emerging applications of metabolomics in drug discovery and precision medicine. Nat. Rev. Drug Discov. 2016, 15, 473-484. [CrossRef]

13. Varella-Garcia, M. Chromosomal and genomic changes in lung cancer. Cell Adhes. Migr. 2010, 4, $100-106$. [CrossRef]

14. Sciacovelli, M.; Gaude, E.; Hilvo, M.; Frezza, C. Chapter One-The Metabolic Alterations of Cancer Cells. In Methods in Enzymology; Galluzzi, L., Kroemer, G., Eds.; Academic Press: Cambridge, MA, USA, 2014; Volume 542, pp. 1-23.

15. Muñoz-Pinedo, C.; El Mjiyad, N.; Ricci, J.E. Cancer metabolism: Current perspectives and future directions. Cell Death Dis. 2012, 3, e248. [CrossRef] [PubMed]

16. Cantor, J.R.; Sabatini, D.M. Cancer Cell Metabolism: One Hallmark, Many Faces. Cancer Discov. 2012,2 , 881. [CrossRef] [PubMed]

17. Callejon-Leblic, B.; Garcia-Barrera, T.; Gravalos-Guzman, J.; Pereira-Vega, A.; Gomez-Ariza, J.L. Metabolic profiling of potential lung cancer biomarkers using bronchoalveolar lavage fluid and the integrated direct infusion/ gas chromatography mass spectrometry platform. J. Proteom. 2016, 145, 197-206. [CrossRef] [PubMed]

18. Fahrmann, J.F.; Kim, K.; DeFelice, B.C.; Taylor, S.L.; Gandara, D.R.; Yoneda, K.Y.; Cooke, D.T.; Fiehn, O.; Kelly, K.; Miyamoto, S. Investigation of metabolomic blood biomarkers for detection of adenocarcinoma lung cancer. Cancer Epidemiol. Biomark. Prev. 2015, 24, 1716-1723. [CrossRef]

19. Cameron, S.J.; Lewis, K.E.; Beckmann, M.; Allison, G.G.; Ghosal, R.; Lewis, P.D.; Mur, L.A. The metabolomic detection of lung cancer biomarkers in sputum. Lung Cancer 2016, 94, 88-95. [CrossRef]

20. Chen, W.; Lu, S.; Ou, J.; Wang, G.; Zu, Y.; Chen, F.; Bai, C. Metabonomic characteristics and biomarker research of human lung cancer tissues by HR1H NMR spectroscopy. Cancer Biomark. 2016, 16, 653-664. [CrossRef]

21. Miyamoto, S.; Taylor, S.L.; Barupal, D.K.; Taguchi, A.; Wohlgemuth, G.; Wikoff, W.R.; Yoneda, K.Y.; Gandara, D.R.; Hanash, S.M.; Kim, K.; et al. Systemic Metabolomic Changes in Blood Samples of Lung Cancer Patients Identified by Gas Chromatography Time-of-Flight Mass Spectrometry. Metabolites 2015, 5, 192-210. [CrossRef]

22. Yu, L.; Li, K.; Zhang, X. Next-generation metabolomics in lung cancer diagnosis, treatment and precision medicine: Mini review. Oncotarget 2017, 8, 115774-115786. [CrossRef]

23. Bamji-Stocke, S.; van Berkel, V.; Miller, D.M.; Frieboes, H.B. A review of metabolism-associated biomarkers in lung cancer diagnosis and treatment. Metabolomics 2018, 14, 81. [CrossRef]

24. Liberati, A.; Altman, D.G.; Tetzlaff, J.; Mulrow, C.; Gøtzsche, P.C.; Ioannidis, J.P.A.; Clarke, M.; Devereaux, P.J.; Kleijnen, J.; Moher, D. The PRISMA statement for reporting systematic reviews and meta-analyses of studies that evaluate healthcare interventions: Explanation and elaboration. BMJ 2009, 339, b2700. [CrossRef] 
25. Pietzke, M.; Arroyo, S.F.; Sumpton, D.; Mackay, G.M.; Martin-Castillo, B.; Camps, J.; Joven, J.; Menendez, J.A.; Vazquez, A. Stratification of cancer and diabetes based on circulating levels of formate and glucose. Cancer Metab. 2019, 7, 3. [CrossRef] [PubMed]

26. Hu, S.; Shen, G.; Yin, S.; Xu, W.; Hu, B. Melatonin and tryptophan circadian profiles in patients with advanced non-small cell lung cancer. Adv. Ther. 2009, 26, 886-892. [CrossRef] [PubMed]

27. Shingyoji, M.; Iizasa, T.; Higashiyama, M.; Imamura, F.; Saruki, N.; Imaizumi, A.; Yamamoto, H.; Daimon, T.; Tochikubo, O.; Mitsushima, T.; et al. The significance and robustness of a plasma free amino acid (PFAA) profile-based multiplex function for detecting lung cancer. BMC Cancer 2013, 13, 77. [CrossRef] [PubMed]

28. Miyagi, Y.; Higashiyama, M.; Gochi, A.; Akaike, M.; Ishikawa, T.; Miura, T.; Saruki, N.; Bando, E.; Kimura, H.; Imamura, F; et al. Plasma free amino acid profiling of five types of cancer patients and its application for early detection. PLoS ONE 2011, 6, e24143. [CrossRef] [PubMed]

29. Kawamoto, H.; Hara, H.; Araya, J.; Ichikawa, A.; Fujita, Y.; Utsumi, H.; Hashimoto, M.; Wakui, H.; Minagawa, S.; Numata, T.; et al. Prostaglandin E-Major Urinary Metabolite (PGE-MUM) as a Tumor Marker for Lung Adenocarcinoma. Cancers (Basel) 2019, 11, 768. [CrossRef]

30. Proenza, A.M.; Oliver, J.; Palou, A.; Roca, P. Breast and lung cancer are associated with a decrease in blood cell amino acid content. J. Nutr. Biochem. 2003, 14, 133-138. [CrossRef]

31. Yue, X.; He, J.; Zhang, R.; Xu, J.; Zhou, Z.; Zhang, R.; Bi, N.; Wang, Z.; Sun, C.; Wang, L.; et al. Biotransformation-based metabolomics profiling method for determining and quantitating cancer-related metabolites. J. Chromatogr. A 2018, 1580, 80-89. [CrossRef]

32. Kanehisa, M.; Goto, S. KEGG: Kyoto encyclopedia of genes and genomes. Nucleic Acids Res. 2000, $28,27-30$. [CrossRef]

33. Kami, K.; Fujimori, T.; Sato, H.; Sato, M.; Yamamoto, H.; Ohashi, Y.; Sugiyama, N.; Ishihama, Y.; Onozuka, H.; Ochiai, A.; et al. Metabolomic profiling of lung and prostate tumor tissues by capillary electrophoresis time-of-flight mass spectrometry. Metabolomics 2013, 9, 444-453. [CrossRef]

34. Preti, G.; Labows, J.N.; Kostelc, J.G.; Aldinger, S.; Daniele, R. Analysis of lung air from patients with bronchogenic carcinoma and controls using gas chromatography-mass spectrometry. J. Chromatogr. B: Biomed. Sci. Appl. 1988, 432, 1-11. [CrossRef]

35. Klupczynska, A.; Plewa, S.; Kasprzyk, M.; Dyszkiewicz, W.; Kokot, Z.J.; Matysiak, J. Serum lipidome screening in patients with stage I non-small cell lung cancer. Clin. Exp. Med. 2019, 19, 505-513. [CrossRef] [PubMed]

36. Ni, J.; Xu, L.; Li, W.; Zheng, C.; Wu, L. Targeted metabolomics for serum amino acids and acylcarnitines in patients with lung cancer. Exp. Ther. Med. 2019, 18, 188-198. [CrossRef] [PubMed]

37. Wan, X.; Wang, W.; Liu, J.; Tong, T. Estimating the sample mean and standard deviation from the sample size, median, range and/or interquartile range. BMC Med. Res. Methodol. 2014, 14, 135. [CrossRef] [PubMed]

38. Wells, G.A.; Shea, B.; O'Connell, D.; Peterson, J.; Welch, V.; Losos, M.; Tugwell, P. The Newcastle-Ottawa Scale (NOS) for Assessing the Quality of Nonrandomised Studies in Meta-Analyses. Available online: http://www.ohri.ca/programs/clinical_epidemiology/oxford.asp (accessed on 18 March 2020).

39. Kukreja, S.C.; Shemerdiak, W.P.; York, P.A.J.; Lad, T.E.; Abramson, E.C.; Thomas, P.A.; Mir, J. Presence of prostaglandin $\mathrm{E}$ in lung tumors from normocalcemic patients. Am. J. Med. 1982, 72, 737-742. [CrossRef]

40. Masri, F.A.; Comhair, S.A.; Koeck, T.; Xu, W.; Janocha, A.; Ghosh, S.; Dweik, R.A.; Golish, J.; Kinter, M.; Stuehr, D.J.; et al. Abnormalities in nitric oxide and its derivatives in lung cancer. Am. J. Respir. Crit. Care Med. 2005, 172, 597-605. [CrossRef]

41. Okur, H.K.; Yuksel, M.; Lacin, T.; Baysungur, V.; Okur, E. Detection of reactive oxygen metabolites in malignant and adjacent normal tissues of patients with lung cancer. World J. Surg. Oncol. 2013, 11, 9. [CrossRef]

42. Ordóñez-Mena, J.M.; Schöttker, B.; Fedirko, V.; Jenab, M.; Olsen, A.; Halkjær, J.; Kampman, E.; de Groot, L.; Jansen, E.; Bueno-de-Mesquita, H.B.; et al. Pre-diagnostic vitamin D concentrations and cancer risks in older individuals: An analysis of cohorts participating in the CHANCES consortium. Eur. J. Epidemiol. 2016, 31, 311-323. [CrossRef]

43. Gao, X.; Wilsgaard, T.; Jansen, E.H.; Holleczek, B.; Zhang, Y.; Xuan, Y.; Anusruti, A.; Brenner, H.; Schottker, B. Pre-diagnostic derivatives of reactive oxygen metabolites and the occurrence of lung, colorectal, breast and prostate cancer: An individual participant data meta-analysis of two large population-based studies. Int. J. Cancer 2019, 145, 49-57. [CrossRef] 
44. Fu, W.; Wang, C.; Zou, L.; Liu, Q.; Gan, Y.; Yan, S.; Song, F.; Wang, Z.; Lu, Z.; Cao, S. Association between exposure to noise and risk of hypertension: A meta-analysis of observational epidemiological studies. J. Hypertens. 2017, 35, 2358-2366. [CrossRef]

45. Higgins, J.; Thomas, J.; Chandler, J.; Cumpston, M.; Li, T.; Page, M.; Welch, V. Cochrane Handbook for Systematic Reviews of Interventions, 2nd ed.; John Wiley \& Sons: Chichester, UK, 2019.

46. DerSimonian, R.; Laird, N. Meta-analysis in clinical trials. Control. Clin. Trials 1986, 7, 177-188. [CrossRef]

47. StataCorp. Stata Statistical Software: Release 14; StataCorp LLC: College Station, TX, USA, 2015.

48. Kilkkinen, A.; Knekt, P.; Heliovaara, M.; Rissanen, H.; Marniemi, J.; Hakulinen, T.; Aromaa, A. Vitamin D status and the risk of lung cancer: A cohort study in Finland. Cancer Epidemiol. Biomark. Prev. 2008, 17, 3274-3278. [CrossRef] [PubMed]

49. Afzal, S.; Bojesen, S.E.; Nordestgaard, B.G. Low Plasma 25-Hydroxyvitamin D and Risk of Tobacco-Related Cancer. Clin. Chem. 2013, 59, 771-780. [CrossRef] [PubMed]

50. de Waard, F.; Kemmeren, J.M.; van Ginkel, L.A.; Stolker, A.A. Urinary cotinine and lung cancer risk in a female cohort. Br. J. Cancer 1995, 72, 784-787. [CrossRef] [PubMed]

51. Ellard, G.A.; de Waard, F.; Kemmeren, J.M. Urinary nicotine metabolite excretion and lung cancer risk in a female cohort. Br. J. Cancer 1995, 72, 788-791. [CrossRef]

52. London, S.J.; Yuan, J.-M.; Chung, F.-L.; Gao, Y.-T.; Coetzee, G.A.; Ross, R.K.; Yu, M.C. Isothiocyanates, glutathione S-transferase M1 and T1 polymorphisms, and lung-cancer risk: A prospective study of men in Shanghai, China. Lancet 2000, 356, 724-729. [CrossRef]

53. Boffetta, P.; Clark, S.; Shen, M.; Gislefoss, R.; Peto, R.; Andersen, A. Serum cotinine level as predictor of lung cancer risk. Cancer Epidemiol. Biomark. Prev. 2006, 15, 1184-1188. [CrossRef]

54. Loft, S.; Svoboda, P.; Kasai, H.; Tjonneland, A.; Moller, P.; Sorensen, M.; Overvad, K.; Autrup, H.; Raaschou-Nielsen, O. Prospective study of urinary excretion of 7-methylguanine and the risk of lung cancer: Effect modification by mu class glutathione-S-transferases. Int. J. Cancer 2007, 121, 1579-1584. [CrossRef]

55. Johansson, M.; Relton, C.; Ueland, P.M.; Vollset, S.E.; Midttun, O.; Nygard, O.; Slimani, N.; Boffetta, P.; Jenab, M.; Clavel-Chapelon, F.; et al. Serum B vitamin levels and risk of lung cancer. JAMA 2010, 303, 2377-2385. [CrossRef]

56. Timofeeva, M.N.; McKay, J.D.; Smith, G.D.; Johansson, M.; Byrnes, G.B.; Chabrier, A.; Relton, C.; Ueland, P.M.; Vollset, S.E.; Midttun, Ø.; et al. Genetic polymorphisms in 15q25 and 19q13 loci, cotinine levels, and risk of lung cancer in EPIC. Cancer Epidemiol. Biomark. Prev. 2011, 20, 2250-2261. [CrossRef]

57. Weinstein, S.J.; Yu, K.; Horst, R.L.; Parisi, D.; Virtamo, J.; Albanes, D. Serum 25-hydroxyvitamin D and risk of lung cancer in male smokers: A nested case-control study. PLOS ONE 2011, 6, e20796. [CrossRef]

58. Yuan, J.M.; Gao, Y.T.; Murphy, S.E.; Carmella, S.G.; Wang, R.; Zhong, Y.; Moy, K.A.; Davis, A.B.; Tao, L.; Chen, M.; et al. Urinary levels of cigarette smoke constituent metabolites are prospectively associated with lung cancer development in smokers. Cancer Res. 2011, 71, 6749-6757. [CrossRef] [PubMed]

59. Yuan, J.M.; Gao, Y.T.; Wang, R.; Chen, M.; Carmella, S.G.; Hecht, S.S. Urinary levels of volatile organic carcinogen and toxicant biomarkers in relation to lung cancer development in smokers. Carcinogenesis 2012, 33, 804-809. [CrossRef] [PubMed]

60. Eom, S.Y.; Yim, D.H.; Moon, S.I.; Youn, J.W.; Kwon, H.J.; Oh, H.C.; Yang, J.J.; Park, S.K.; Yoo, K.Y.; Kim, H.S.; et al. Polycyclic aromatic hydrocarbon-induced oxidative stress, antioxidant capacity, and the risk of lung cancer: A pilot nested case-control study. Anticancer Res. 2013, 33, 3089-3097.

61. Chuang, S.C.; Fanidi, A.; Ueland, P.M.; Relton, C.; Midttun, O.; Vollset, S.E.; Gunter, M.J.; Seckl, M.J.; Travis, R.C.; Wareham, N.; et al. Circulating biomarkers of tryptophan and the kynurenine pathway and lung cancer risk. Cancer Epidemiol. Biomark. Prev. 2014, 23, 461-468. [CrossRef] [PubMed]

62. Mathe, E.A.; Patterson, A.D.; Haznadar, M.; Manna, S.K.; Krausz, K.W.; Bowman, E.D.; Shields, P.G.; Idle, J.R.; Smith, P.B.; Anami, K.; et al. Noninvasive urinary metabolomic profiling identifies diagnostic and prognostic markers in lung cancer. Cancer Res. 2014, 74, 3259-3270. [CrossRef] [PubMed]

63. Yuan, J.M.; Butler, L.M.; Gao, Y.T.; Murphy, S.E.; Carmella, S.G.; Wang, R.; Nelson, H.H.; Hecht, S.S. Urinary metabolites of a polycyclic aromatic hydrocarbon and volatile organic compounds in relation to lung cancer development in lifelong never smokers in the Shanghai Cohort Study. Carcinogenesis 2014, 35, 339-345. [CrossRef] 
64. Wang, X.; Cui, J.; Gu, J.; He, H.; Li, B.; Li, W. Plasma 25-hydroxyvitamin D deficiency is associated with the risk of non-small cell lung cancer in a Chinese population. Cancer Biomark. 2015, 15, 663-668. [CrossRef]

65. Haznadar, M.; Cai, Q.; Krausz, K.W.; Bowman, E.D.; Margono, E.; Noro, R.; Thompson, M.D.; Mathe, E.A.; Munro, H.M.; Steinwandel, M.D.; et al. Urinary Metabolite Risk Biomarkers of Lung Cancer: A Prospective Cohort Study. Cancer Epidemiol. Biomark. Prev. 2016, 25, 978-986. [CrossRef]

66. Yuan, J.M.; Nelson, H.H.; Butler, L.M.; Carmella, S.G.; Wang, R.; Kuriger-Laber, J.K.; Adams-Haduch, J.; Hecht, S.S.; Gao, Y.T.; Murphy, S.E. Genetic determinants of cytochrome P450 2A6 activity and biomarkers of tobacco smoke exposure in relation to risk of lung cancer development in the Shanghai cohort study. Int. J. Cancer 2016, 138, 2161-2171. [CrossRef]

67. Yuan, J.M.; Nelson, H.H.; Carmella, S.G.; Wang, R.; Kuriger-Laber, J.; Jin, A.; Adams-Haduch, J.; Hecht, S.S.; Koh, W.P.; Murphy, S.E. CYP2A6 genetic polymorphisms and biomarkers of tobacco smoke constituents in relation to risk of lung cancer in the Singapore Chinese Health Study. Carcinogenesis 2017, 38, 411-418. [CrossRef]

68. Fanidi, A.; Muller, D.C.; Yuan, J.-M.; Stevens, V.L.; Weinstein, S.J.; Albanes, D.; Prentice, R.; Thomsen, C.A.; Pettinger, M.; Cai, Q.; et al. Circulating Folate, Vitamin B6, and Methionine in Relation to Lung Cancer Risk in the Lung Cancer Cohort Consortium (LC3). J. Nat. Cancer Inst. 2018, 110, 57-67. [CrossRef]

69. Haznadar, M.; Krausz, K.W.; Margono, E.; Diehl, C.M.; Bowman, E.D.; Manna, S.K.; Robles, A.I.; Ryan, B.M.; Gonzalez, F.J.; Harris, C.C. Inverse association of vitamin D3 levels with lung cancer mediated by genetic variation. Cancer Med. 2018, 7, 2764-2775. [CrossRef]

70. Larose, T.L.; Guida, F.; Fanidi, A.; Langhammer, A.; Kveem, K.; Stevens, V.L.; Jacobs, E.J.; Smith-Warner, S.A.; Giovannucci, E.; Albanes, D.; et al. Circulating cotinine concentrations and lung cancer risk in the Lung Cancer Cohort Consortium (LC3). Int. J. Epidemiol. 2018, 47, 1760-1771. [CrossRef] [PubMed]

71. Gao, X.; Xuan, Y.; Benner, A.; Anusruti, A.; Brenner, H.; Schottker, B. Nitric Oxide Metabolites and Lung Cancer Incidence: A Matched Case-Control Study Nested in the ESTHER Cohort. Oxidative Med. Cell. Longev. 2019, 2019, 6470950. [CrossRef] [PubMed]

72. Seow, W.J.; Shu, X.O.; Nicholson, J.K.; Holmes, E.; Walker, D.I.; Hu, W.; Cai, Q.; Gao, Y.T.; Xiang, Y.B.; Moore, S.C.; et al. Association of Untargeted Urinary Metabolomics and Lung Cancer Risk Among Never-Smoking Women in China. JAMA Netw. Open 2019, 2, e1911970. [CrossRef] [PubMed]

73. Hendrick, A.M.; Mitchell, M.D.; Harris, A.L. Plasma prostaglandins in lung cancer. Eur. J. Cancer Clin. Oncol. 1988, 24, 1069-1071. [CrossRef]

74. Gencer, M.; Ceylan, E.; Aksoy, N.; Uzun, K. Association of serum reactive oxygen metabolite levels with different histopathological types of lung cancer. Respiration 2006, 73, 520-524. [CrossRef]

75. Zhang, T.; Lai, B.; Duan, L.; Wang, X. [Detection of metabolites of tobacco-specific nitrosamine 4-(methylnitrosamino)-1-(3-pyridyl)-1-butanone in lung cancer smokers' urine]. Zhongguo Fei Ai Za Zhi 2006, 9, 231-235. [CrossRef]

76. Esme, H.; Cemek, M.; Sezer, M.; Saglam, H.; Demir, A.; Melek, H.; Unlu, M. High levels of oxidative stress in patients with advanced lung cancer. Respirology 2008, 13, 112-116. [CrossRef]

77. Hwang, S.H.; Ryu, H.J.; Kang, S.J.; Yun, E.H.; Lim, M.K.; Kim, H.T.; Lee, J.S.; Lee, D.H. Levels of tobacco-specific metabolites among non-smoking lung cancer cases at diagnosis: Case-control findings. Asian Pac. J. Cancer Prev. 2014, 14, 6591-6593. [CrossRef]

78. Kim, H.J.; Jang, S.H.; Ryu, J.-S.; Lee, J.E.; Kim, Y.C.; Lee, M.K.; Jang, T.W.; Lee, S.-Y.; Nakamura, H.; Nishikata, N.; et al. The performance of a novel amino acid multivariate index for detecting lung cancer: A case control study in Korea. Lung Cancer 2015, 90, 522-527. [CrossRef] [PubMed]

79. Klupczynska, A.; Plewa, S.; Dyszkiewicz, W.; Kasprzyk, M.; Sytek, N.; Kokot, Z.J. Determination of low-molecular-weight organic acids in non-small cell lung cancer with a new liquid chromatography-tandem mass spectrometry method. J. Pharm Biomed. Anal. 2016, 129, 299-309. [CrossRef] [PubMed]

80. Klupczynska, A.; Derezinski, P.; Dyszkiewicz, W.; Pawlak, K.; Kasprzyk, M.; Kokot, Z.J. Evaluation of serum amino acid profiles' utility in non-small cell lung cancer detection in Polish population. Lung Cancer 2016, 100, 71-76. [CrossRef] [PubMed]

81. Ni, J.; Xu, L.; Li, W.; Wu, L. Simultaneous determination of thirteen kinds of amino acid and eight kinds of acylcarnitine in human serum by LC-MS/MS and its application to measure the serum concentration of lung cancer patients. Biomed. Chromatogr. 2016, 30, 1796-1806. [CrossRef] 
82. Zhang, Q.; Xu, H.; Liu, R.; Gao, P.; Yang, X.; Jin, W.; Zhang, Y.; Bi, K.; Li, Q. A Novel Strategy for Targeted Lipidomics Based on LC-Tandem-MS Parameters Prediction, Quantification, and Multiple Statistical Data Mining: Evaluation of Lysophosphatidylcholines as Potential Cancer Biomarkers. Anal. Chem. 2019, 91, 3389-3396. [CrossRef]

83. Church, T.R.; Anderson, K.E.; Caporaso, N.E.; Geisser, M.S.; Le, C.T.; Zhang, Y.; Benoit, A.R.; Carmella, S.G.; Hecht, S.S. A prospectively measured serum biomarker for a tobacco-specific carcinogen and lung cancer in smokers. Cancer Epidemiol. Biomark. Prev. 2009, 18, 260-266. [CrossRef]

84. Skaaby, T.; Husemoen, L.L.; Thuesen, B.H.; Pisinger, C.; Jorgensen, T.; Roswall, N.; Larsen, S.C.; Linneberg, A. Prospective population-based study of the association between serum 25-hydroxyvitamin-D levels and the incidence of specific types of cancer. Cancer Epidemiol. Biomark. Prev. 2014, 23, 1220-1229. [CrossRef]

85. Maeda, J.; Higashiyama, M.; Imaizumi, A.; Nakayama, T.; Yamamoto, H.; Daimon, T.; Yamakado, M.; Imamura, F.; Kodama, K. Possibility of multivariate function composed of plasma amino acid profiles as a novel screening index for non-small cell lung cancer: A case control study. BMC Cancer 2010, 10, 690. [CrossRef]

86. Yuan, J.M.; Koh, W.P.; Murphy, S.E.; Fan, Y.; Wang, R.; Carmella, S.G.; Han, S.; Wickham, K.; Gao, Y.T.; Yu, M.C.; et al. Urinary levels of tobacco-specific nitrosamine metabolites in relation to lung cancer development in two prospective cohorts of cigarette smokers. Cancer Res. 2009, 69, 2990-2995. [CrossRef]

87. Hecht, S.S.; Murphy, S.E.; Stepanov, I.; Nelson, H.H.; Yuan, J.M. Tobacco smoke biomarkers and cancer risk among male smokers in the Shanghai cohort study. Cancer Lett. 2013, 334, 34-38. [CrossRef]

88. Lai, H.-S.; Lee, J.-C.; Lee, P.-H.; Wang, S.-T.; Chen, W.-J. Plasma free amino acid profile in cancer patients. Semi. Cancer Biol. 2005, 15, 267-276. [CrossRef] [PubMed]

89. Hashim, N.A.A.; Ab-Rahim, S.; Suddin, L.S.; Saman, M.S.A.; Mazlan, M. Global serum metabolomics profiling of colorectal cancer. Mol. Clin. Oncol. 2019, 11, 3-14. [CrossRef] [PubMed]

90. Yusof, H.M.; Ab-Rahim, S.; Suddin, L.S.; Saman, M.S.A.; Mazlan, M. Metabolomics Profiling on Different Stages of Colorectal Cancer: A Systematic Review. Malays. J. Med. Sci. 2018, 25, 16-34. [CrossRef] [PubMed]

91. Chen, Z.; Li, Z.; Li, H.; Jiang, Y. Metabolomics: A promising diagnostic and therapeutic implement for breast cancer. OncoTargets Ther. 2019, 12, 6797-6811. [CrossRef] [PubMed]

92. Norton, J.A.; Gorschboth, C.M.; Wesley, R.A.; Burt, M.E.; Brennan, M.F. Fasting plasma amino acid levels in cancer patients. Cancer 1985, 56, 1181-1186. [CrossRef]

93. Kubota, A.; Meguid, M.M.; Hitch, D.C. Amino acid profiles correlate diagnostically with organ site in three kinds of malignant tumors. Cancer 1992, 69, 2343-2348. [CrossRef]

94. Cascino, A.; Muscaritoli, M.; Cangiano, C.; Conversano, L.; Laviano, A.; Ariemma, S.; Meguid, M.M.; Rossi Fanelli, F. Plasma amino acid imbalance in patients with lung and breast cancer. Anticancer Res. 1995, 15, 507-510.

95. Georgiannos, S.N.; Weston, P.M.; Goode, A.W. Correlation between albuminuria and positively charged amino acids in gastrointestinal cancer. Int. Surg. 1995, 80, 49-52.

96. Sugimura, T.; Birnbaum, S.M.; Winitz, M.; Greenstein, J.P. Quantitative nutritional studies with water-soluble, chemically defined diets. VIII. The forced feeding of diets each lacking in one essential amino acid. Arch. Biochem. Biophys. 1959, 81, 448-455. [CrossRef]

97. Halpern, B.C.; Clark, B.R.; Hardy, D.N.; Halpern, R.M.; Smith, R.A. The effect of replacement of methionine by homocystine on survival of malignant and normal adult mammalian cells in culture. Proc. Natl. Acad. Sci. USA 1974, 71, 1133-1136. [CrossRef]

98. Mecham, J.O.; Rowitch, D.; Wallace, C.D.; Stern, P.H.; Hoffman, R.M. The metabolic defect of methionine dependence occurs frequently in human tumor cell lines. Biochem. Biophys. Res. Commun. 1983, 117, 429-434. [CrossRef]

99. Stern, P.H.; Wallace, C.D.; Hoffman, R.M. Altered methionine metabolism occurs in all members of a set of diverse human tumor cell lines. J. Cell. Physiol. 1984, 119, 29-34. [CrossRef] [PubMed]

100. Lieu, E.L.; Nguyen, T.; Rhyne, S.; Kim, J. Amino acids in cancer. Exp. Mol. Med. 2020, 52, 15-30. [CrossRef] [PubMed]

101. Locasale, J.W. Serine, glycine and one-carbon units: Cancer metabolism in full circle. Nat. Rev. Cancer 2013, 13, 572-583. [CrossRef] [PubMed] 
102. Shuvalov, O.; Petukhov, A.; Daks, A.; Fedorova, O.; Vasileva, E.; Barlev, N.A. One-carbon metabolism and nucleotide biosynthesis as attractive targets for anticancer therapy. Oncotarget 2017, 8, 23955-23977. [CrossRef]

103. Clemens, M.J.; Elia, A.; Morley, S.J. Regulation and dysregulation of protein synthesis in cancer cells. In Systems Biology of Cancer; Thiagalingam, S., Ed.; Cambridge University Press: Cambridge, UK, 2015; pp. 70-92. [CrossRef]

104. Borrego, S.L.; Fahrmann, J.; Datta, R.; Stringari, C.; Grapov, D.; Zeller, M.; Chen, Y.; Wang, P.; Baldi, P.; Gratton, E.; et al. Metabolic changes associated with methionine stress sensitivity in MDA-MB-468 breast cancer cells. Cancer Metab. 2016, 4, 9. [CrossRef]

105. Booher, K.; Lin, D.-W.; Borrego, S.L.; Kaiser, P. Downregulation of Cdc6 and pre-replication complexes in response to methionine stress in breast cancer cells. Cell Cycle (Georgetown, Tex.) 2012, 11, 4414-4423. [CrossRef]

106. Tsou, J.A.; Hagen, J.A.; Carpenter, C.L.; Laird-Offringa, I.A. DNA methylation analysis: A powerful new tool for lung cancer diagnosis. Oncogene 2002, 21, 5450-5461. [CrossRef]

107. Shi, Y.X.; Sheng, D.Q.; Cheng, L.; Song, X.Y. Current Landscape of Epigenetics in Lung Cancer: Focus on the Mechanism and Application. J. Oncol. 2019, 2019, 8107318. [CrossRef]

108. Gupta, N.; Miyauchi, S.; Martindale, R.G.; Herdman, A.V.; Podolsky, R.; Miyake, K.; Mager, S.; Prasad, P.D.; Ganapathy, M.E.; Ganapathy, V. Upregulation of the amino acid transporter ATB0,+ (SLC6A14) in colorectal cancer and metastasis in humans. Biochim. Biophys. Acta (BBA)-Mol. Basis Dis. 2005, 1741, 215-223. [CrossRef]

109. Gupta, N.; Prasad, P.D.; Ghamande, S.; Moore-Martin, P.; Herdman, A.V.; Martindale, R.G.; Podolsky, R.; Mager, S.; Ganapathy, M.E.; Ganapathy, V. Up-regulation of the amino acid transporter ATB ${ }^{0,+}$ (SLC6A14) in carcinoma of the cervix. Gynecol. Oncol. 2006, 100, 8-13. [CrossRef] [PubMed]

110. Karunakaran, S.; Umapathy, N.S.; Thangaraju, M.; Hatanaka, T.; Itagaki, S.; Munn, D.H.; Prasad, P.D.; Ganapathy, V. Interaction of tryptophan derivatives with SLC6A14 $($ ATB0,+) reveals the potential of the transporter as a drug target for cancer chemotherapy. Biochem. J. 2008, 414, 343-355. [CrossRef] [PubMed]

111. Lu, S.C. Glutathione synthesis. Biochim. Biophys. Acta (BBA)-Bioenerg. 2013, 1830, 3143-3153. [CrossRef]

112. Janssen-Heininger, Y.M.; Nolin, J.D.; Hoffman, S.M.; van der Velden, J.L.; Tully, J.E.; Lahue, K.G.; Abdalla, S.T.; Chapman, D.G.; Reynaert, N.L.; van der Vliet, A.; et al. Emerging mechanisms of glutathione-dependent chemistry in biology and disease. J. Cell. Biochem. 2013, 114, 1962-1968. [CrossRef] [PubMed]

113. Chai, E.Z.; Siveen, K.S.; Shanmugam, M.K.; Arfuso, F.; Sethi, G. Analysis of the intricate relationship between chronic inflammation and cancer. Biochem. J. 2015, 468, 1-15. [CrossRef]

114. Campbell, K.; Vowinckel, J.; Keller, M.A.; Ralser, M. Methionine Metabolism Alters Oxidative Stress Resistance via the Pentose Phosphate Pathway. Antioxid. Redox Signal. 2016, 24, 543-547. [CrossRef]

115. Luo, S.; Levine, R.L. Methionine in proteins defends against oxidative stress. FASEB J. 2009, 23, 464-472. [CrossRef]

116. van Baren, N.; Van den Eynde, B.J. Tryptophan-Degrading Enzymes in Tumoral Immune Resistance. Front. Immunol. 2016, 6. [CrossRef]

117. Badawy, A.A.B. Kynurenine Pathway of Tryptophan Metabolism: Regulatory and Functional Aspects. Int. J. Tryptophan Res. 2017, 10, 1178646917691938. [CrossRef]

118. Munn, D.H.; Mellor, A.L. Indoleamine 2,3-dioxygenase and tumor-induced tolerance. J. Clin. Investig. 2007, 117, 1147-1154. [CrossRef]

119. Heng, B.; Lim, C.K.; Lovejoy, D.B.; Bessede, A.; Gluch, L.; Guillemin, G.J. Understanding the role of the kynurenine pathway in human breast cancer immunobiology. Oncotarget 2016, 7, 6506-6520. [CrossRef] [PubMed]

120. Phang, J.M. Proline Metabolism in Cell Regulation and Cancer Biology: Recent Advances and Hypotheses. Antioxid. Redox Signal. 2017, 30, 635-649. [CrossRef] [PubMed]

121. Nagano, T.; Nakano, M.; Nakashima, A.; Onishi, K.; Yamao, S.; Enari, M.; Kikkawa, U.; Kamada, S. Identification of cellular senescence-specific genes by comparative transcriptomics. Sci. Rep. 2016, 6, 31758. [CrossRef]

122. Nagano, T.; Nakashima, A.; Onishi, K.; Kawai, K.; Awai, Y.; Kinugasa, M.; Iwasaki, T.; Kikkawa, U.; Kamada, S. Proline dehydrogenase promotes senescence through the generation of reactive oxygen species. J. Cell Sci. 2017, 130, 1413-1420. [CrossRef] [PubMed] 
123. Liu, Y.; Mao, C.; Wang, M.; Liu, N.; Ouyang, L.; Liu, S.; Tang, H.; Cao, Y.; Liu, S.; Wang, X.; et al. Cancer progression is mediated by proline catabolism in non-small cell lung cancer. Oncogene 2020, 39, 2358-2376. [CrossRef]

124. Hecht, S.S. Tobacco smoke carcinogens and lung cancer. J. Natl. Cancer Inst. 1999, 91, 1194-1210. [CrossRef]

125. Hecht, S.S. Lung carcinogenesis by tobacco smoke. Int. J.Cancer 2012, 131, 2724-2732. [CrossRef]

126. Tobacco smoke and involuntary smoking. IARC Monogr. Eval. Carcinog. Risks Hum. 2004, 83, 1-1438.

127. West, R. Tobacco smoking: Health impact, prevalence, correlates and interventions. Psychol. Health 2017, 32, 1018-1036. [CrossRef]

128. Thielen, A.; Klus, H.; Muller, L. Tobacco smoke: Unraveling a controversial subject. Exp. Toxicol. Pathol. 2008, 60, 141-156. [CrossRef]

129. Borgerding, M.; Klus, H. Analysis of complex mixtures-cigarette smoke. Exp. Toxicol. Pathol. 2005, 57 (Suppl. 1), 43-73. [CrossRef]

130. Hecht, S.S. Research opportunities related to establishing standards for tobacco products under the Family Smoking Prevention and Tobacco Control Act. Nicotine Tob. Res. 2012, 14, 18-28. [CrossRef] [PubMed]

131. Benowitz, N.L.; Hukkanen, J.; Jacob, P. Nicotine chemistry, metabolism, kinetics and biomarkers. In Handbook of Experimental Pharmacology; Springer: Berlin, Germany, 2009. [CrossRef]

132. Jacob, P.; Shulgin, A.T.; Benowitz, N.L. Synthesis of (3'R,5'S)-trans-3'-hydroxycotinine, a major metabolite of nicotine. Metabolic formation of 3'-hydroxycotinine in humans is highly stereoselective. J. Med. Chem. 1990, 33, 1888-1891. [CrossRef] [PubMed]

133. Neurath, G.B.; Dünger, M.; Orth, D.; Pein, F.G. Trans-3'-hydroxycotinine as a main metabolite in urine of smokers. Int. Arch. Occup. Environ. Health 1987, 59, 199-201. [CrossRef] [PubMed]

134. Murphy, S.E.; von Weymarn, L.B.; Schutten, M.M.; Kassie, F.; Modiano, J.F. Chronic nicotine consumption does not influence 4-(methylnitrosamino)-1-(3-pyridyl)-1-butanone-induced lung tumorigenesis. Cancer Prev. Res. (Phila) 2011, 4, 1752-1760. [CrossRef] [PubMed]

135. Maier, C.R.; Hollander, M.C.; Hobbs, E.A.; Dogan, I.; Linnoila, R.I.; Dennis, P.A. Nicotine does not enhance tumorigenesis in mutant K-ras-driven mouse models of lung cancer. Cancer Prev. Res. (Phila) 2011, 4, 1743-1751. [CrossRef]

136. Benowitz, N.L.; Dains, K.M.; Dempsey, D.; Yu, L.; Jacob, P., 3rd. Estimation of nicotine dose after low-level exposure using plasma and urine nicotine metabolites. Cancer Epidemiol. Biomark. Prev. 2010, 19, 1160-1166. [CrossRef]

137. Benowitz, N.L.; St. Helen, G.; Nardone, N.; Cox, L.S.; Jacob, P., III. Urine Metabolites for Estimating Daily Intake of Nicotine From Cigarette Smoking. Nicotine Tob Res. 2019, 22, 288-292. [CrossRef]

138. Moorthy, B.; Chu, C.; Carlin, D.J. Polycyclic aromatic hydrocarbons: From metabolism to lung cancer. Toxicol. Sci. 2015, 145, 5-15. [CrossRef]

139. Singh, A.; Kamal, R.; Ahamed, I.; Wagh, M.; Bihari, V.; Sathian, B.; Kesavachandran, C.N. PAH exposure-associated lung cancer: An updated meta-analysis. Occup. Med. 2018, 68, 255-261. [CrossRef]

140. Armstrong, B.; Hutchinson, E.; Unwin, J.; Fletcher, T. Lung cancer risk after exposure to polycyclic aromatic hydrocarbons: A review and meta-analysis. Environ. Health Perspect. 2004, 112, 970-978. [CrossRef] [PubMed]

141. Bostrom, C.E.; Gerde, P.; Hanberg, A.; Jernstrom, B.; Johansson, C.; Kyrklund, T.; Rannug, A.; Tornqvist, M.; Victorin, K.; Westerholm, R. Cancer risk assessment, indicators, and guidelines for polycyclic aromatic hydrocarbons in the ambient air. Environ. Health Perspect. 2002, 110, 451-488. [CrossRef] [PubMed]

142. Some non-heterocyclic polycyclic aromatic hydrocarbons and some related exposures. IARC Monogr. Eval. Carcinog. Risks Hum. 2010, 92, 1-853.

143. Miller, K.P.; Ramos, K.S. Impact of cellular metabolism on the biological effects of benzo[a]pyrene and related hydrocarbons. Drug Metab. Rev. 2001, 33, 1-35. [CrossRef]

144. Ramos, K.S.; Moorthy, B. Bioactivation of polycyclic aromatic hydrocarbon carcinogens within the vascular wall: Implications for human atherogenesis. Drug Metab. Rev. 2005, 37, 595-610. [CrossRef]

145. Rybicki, B.A.; Nock, N.L.; Savera, A.T.; Tang, D.; Rundle, A. Polycyclic aromatic hydrocarbon-DNA adduct formation in prostate carcinogenesis. Cancer Lett. 2006, 239, 157-167. [CrossRef]

146. Alexandrov, K.; Rojas, M.; Satarug, S. The critical DNA damage by benzo(a)pyrene in lung tissues of smokers and approaches to preventing its formation. Toxicol. Lett. 2010, 198, 63-68. [CrossRef] 
147. Hecht, S.S.; Chen, M.; Yagi, H.; Jerina, D.M.; Carmella, S.G. r-1,t-2,3,c-4-Tetrahydroxy-1,2, 3,4-tetrahydrophenanthrene in Human Urine. Cancer Epidemiol. Biomark. Prev. 2003, 12, 1501.

148. Gunduz, I.; Kondylis, A.; Jaccard, G.; Renaud, J.M.; Hofer, R.; Ruffieux, L.; Gadani, F. Tobacco-specific N-nitrosamines NNN and NNK levels in cigarette brands between 2000 and 2014. Regul. Toxicol. Pharmacol. 2016, 76, 113-120. [CrossRef]

149. Yalcin, E.; de la Monte, S. Tobacco nitrosamines as culprits in disease: Mechanisms reviewed. J. Physiol. Biochem. 2016, 72, 107-120. [CrossRef]

150. Chang, C.M.; Edwards, S.H.; Arab, A.; Del Valle-Pinero, A.Y.; Yang, L.; Hatsukami, D.K. Biomarkers of Tobacco Exposure: Summary of an FDA-Sponsored Public Workshop. Cancer Epidemiol. Biomark. Prev. 2017, 26, 291-302. [CrossRef] [PubMed]

151. Hecht, S.S.; Yuan, J.-M.; Hatsukami, D. Applying tobacco carcinogen and toxicant biomarkers in product regulation and cancer prevention. Chem. Res. Toxicol. 2010, 23, 1001-1008. [CrossRef] [PubMed]

152. Bagchi, P.; Geldner, N.; deCastro, B.R.; De Jesús, V.R.; Park, S.K.; Blount, B.C. Crotonaldehyde exposure in U.S. tobacco smokers and nonsmokers: NHANES 2005-2006 and 2011-2012. Environ. Res. 2018, 163, 1-9. [CrossRef] [PubMed]

153. Chung, F.L.; Tanaka, T.; Hecht, S.S. Induction of liver tumors in F344 rats by crotonaldehyde. Cancer Res. 1986, 46, 1285-1289.

154. International Agency for Research on Cancer, WHO. IARC Monographs on the Identification of Carcinogenic Hazards to Humans. Available online: https://monographs.iarc.fr/list-of-classifications/ (accessed on 1 April 2020).

155. Talhout, R.; Schulz, T.; Florek, E.; van Benthem, J.; Wester, P.; Opperhuizen, A. Hazardous compounds in tobacco smoke. Int. J. Environ. Res. Public Health 2011, 8, 613-628. [CrossRef]

156. Nazaroff, W.W.; Singer, B.C. Inhalation of hazardous air pollutants from environmental tobacco smoke in US residences. J. Expo. Anal. Environ. Epidemiol. 2004, 14, S71-S77. [CrossRef]

157. Wang, H.-T.; Hu, Y.; Tong, D.; Huang, J.; Gu, L.; Wu, X.-R.; Chung, F.-L.; Li, G.-M.; Tang, M.-s. Effect of carcinogenic acrolein on DNA repair and mutagenic susceptibility. J. Biol. Chem. 2012, 287, 12379-12386. [CrossRef]

158. Huff, J. Benzene-induced cancers: Abridged history and occupational health impact. Int. J. Occup. Environ. Health 2007, 13, 213-221. [CrossRef]

159. Baan, R.; Grosse, Y.; Straif, K.; Secretan, B.; El Ghissassi, F.; Bouvard, V.; Benbrahim-Tallaa, L.; Guha, N.; Freeman, C.; Galichet, L.; et al. A review of human carcinogens-Part F: Chemical agents and related occupations. Lancet Oncol. 2009, 10, 1143-1144. [CrossRef]

160. Krolikowski, F.J.; Reuter, K.; Waalkes, T.P.; Sieber, S.M.; Adamson, R.H. Serum sialic acid levels in lung cancer patients. Pharmacology 1976, 14, 47-51. [CrossRef]

161. Hogan-Ryan, A.; Fennelly, J.J.; Jones, M.; Cantwell, B.; Duffy, M.J. Serum sialic acid and CEA concentrations in human breast cancer. Br. J. Cancer 1980, 41, 587-592. [CrossRef] [PubMed]

162. Patel, P.S.; Adhvaryu, S.G.; Balar, D.B.; Parikh, B.J.; Shah, P.M. Clinical application of serum levels of sialic acid, fucose and seromucoid fraction as tumour markers in human leukemias. Anticancer Res. 1994, 14, 747-751. [PubMed]

163. Feijoo, C.; Paez de la Cadena, M.; Rodriguez-Berrocal, F.J.; Martinez-Zorzano, V.S. Sialic acid levels in serum and tissue from colorectal cancer patients. Cancer Lett. 1997, 112, 155-160. [CrossRef]

164. Berbec, H.; Paszkowska, A.; Siwek, B.; Gradziel, K.; Cybulski, M. Total serum sialic acid concentration as a supporting marker of malignancy in ovarian neoplasia. Eur. J. Gynaecol. Oncol. 1999, 20, 389-392.

165. Wongkham, S.; Boonla, C.; Kongkham, S.; Wongkham, C.; Bhudhisawasdi, V.; Sripa, B. Serum total sialic acid in cholangiocarcinoma patients: An ROC curve analysis. Clin. Biochem. 2001, 34, 537-541. [CrossRef]

166. Zhang, Z.; Wuhrer, M.; Holst, S. Serum sialylation changes in cancer. Glycoconj. J. 2018, 35, $139-160$. [CrossRef]

167. Helenius, A.; Aebi, M. Intracellular Functions of N-Linked Glycans. Science 2001, 291, 2364. [CrossRef]

168. Rudd, P.M.; Wormald, M.R.; Stanfield, R.L.; Huang, M.; Mattsson, N.; Speir, J.A.; DiGennaro, J.A.; Fetrow, J.S.; Dwek, R.A.; Wilson, I.A. Roles for glycosylation of cell surface receptors involved in cellular immune recognition. J. Mol. Biol. 1999, 293, 351-366. [CrossRef]

169. Vajaria, B.N.; Patel, P.S. Glycosylation: A hallmark of cancer? Glycoconj. J. 2017, 34, 147-156. [CrossRef] 
170. Shah, M.H.; Telang, S.D.; Shah, P.M.; Patel, P.S. Tissue and serum $\alpha 2-3$ - and $\alpha 2-6$-linkage specific sialylation changes in oral carcinogenesis. Glycoconj. J. 2008, 25, 279-290. [CrossRef]

171. Fuster, M.M.; Esko, J.D. The sweet and sour of cancer: Glycans as novel therapeutic targets. Nat. Rev. Cancer 2005, 5, 526-542. [CrossRef] [PubMed]

172. Yin, L.; Ordonez-Mena, J.M.; Chen, T.; Schottker, B.; Arndt, V.; Brenner, H. Circulating 25-hydroxyvitamin D serum concentration and total cancer incidence and mortality: A systematic review and meta-analysis. Prev. Med. 2013, 57, 753-764. [CrossRef] [PubMed]

173. Chen, G.C.; Zhang, Z.L.; Wan, Z.; Wang, L.; Weber, P.; Eggersdorfer, M.; Qin, L.Q.; Zhang, W. Circulating 25-hydroxyvitamin D and risk of lung cancer: A dose-response meta-analysis. Cancer Causes Control. 2015, 26, 1719-1728. [CrossRef] [PubMed]

174. Lu, W.; Su, X.; Klein, M.S.; Lewis, I.A.; Fiehn, O.; Rabinowitz, J.D. Metabolite Measurement: Pitfalls to Avoid and Practices to Follow. Ann. Rev. Biochem. 2017, 86, 277-304. [CrossRef]

175. Sumner, L.W.; Amberg, A.; Barrett, D.; Beale, M.H.; Beger, R.; Daykin, C.A.; Fan, T.W.M.; Fiehn, O.; Goodacre, R.; Griffin, J.L.; et al. Proposed minimum reporting standards for chemical analysis. Metabolomics 2007, 3, 211-221. [CrossRef]

176. Janssen-Heijnen, M.L.; Schipper, R.M.; Razenberg, P.P.; Crommelin, M.A.; Coebergh, J.W. Prevalence of co-morbidity in lung cancer patients and its relationship with treatment: A population-based study. Lung Cancer 1998, 21, 105-113. [CrossRef]

177. López-Encuentra, A. Comorbidity in operable lung cancer: A multicenter descriptive study on 2992 patients. Lung Cancer 2002, 35, 263-269. [CrossRef]

178. Dima, S.; Chen, K.-H.; Wang, K.-J.; Wang, K.-M.; Teng, N.-C. Effect of Comorbidity on Lung Cancer Diagnosis Timing and Mortality: A Nationwide Population-Based Cohort Study in Taiwan. BioMed Res. Int. 2018, 2018, 1252897. [CrossRef]

179. Ran, N.; Pang, Z.; Gu, Y.; Pan, H.; Zuo, X.; Guan, X.; Yuan, Y.; Wang, Z.; Guo, Y.; Cui, Z.; et al. An Updated Overview of Metabolomic Profile Changes in Chronic Obstructive Pulmonary Disease. Metabolites 2019, 9 , 111. [CrossRef]

180. Arneth, B.; Arneth, R.; Shams, M. Metabolomics of Type 1 and Type 2 Diabetes. Int. J. Mol. Sci. 2019, 20, 2467. [CrossRef]

(C) 2020 by the authors. Licensee MDPI, Basel, Switzerland. This article is an open access article distributed under the terms and conditions of the Creative Commons Attribution (CC BY) license (http://creativecommons.org/licenses/by/4.0/). 